\title{
First Integrals and the Residual Solution for Orthotropic Plates in Plane Strain or Axisymmetric Deformations
}

\author{
By Yihan Lin* and Frederic Y. M. Wan+
}

Two classes of exact solutions are derived for the equations of three dimensional linear orthotropic elasticity theory governing flat (plate) bodies in plane strain or axisymmetric deformations. One of these is the analogue of the Lévy solution for plane strain deformations of isotropic plates and is designated as the interior solutions. The other complementary class correspond to the Papkovich-Fadle Eigenfunction solutions for isotropic rectangular strips and is designated as the residual solutions. For sufficiently thin plates, the latter exhibits rapid exponential decay away from the plate edges. A set of first integrals of the elasticity equations is also derived. These first integrals are then transformed into a set of exact necessary conditions for the elastostatic state of the body to be a residual state. The results effectively remove the asymptoticity restriction of rapid exponential decay of the residual state inherent in the corresponding necessary conditions for isotropic plate problems. The requirement of rapid exponential decay effectively limits their applicability to thin plates. The result of the present paper extend the known results to thick plate problems and to orthotropic plate problems. They enable us to formulate the correct edge conditions for twodimensional orthotropic thick plate theories with stress or mixed edge data.

\section{I ntroduction}

Even before the inception of the three-dimensional theory of elasticity in the nineteenth century, approximate descriptions of the behavior of slender and thin

Address for correspondence: Professor F. Y. M. Wan, Department of Applied Mathematics, University of Washington, FS-20, Seattle, WA 98195 
elastic bodies were formulated in terms of appropriate boundary value problems in one- or two-spatial dimensions. From $[1,3,4,18,19,20]$, we now know that the classical Germain-Kirchhoff two-dimensional linear theory for thin plates [2, 11] corresponds to the leading term of the outer asymptotic expansion of the exact elasticity solution. It is characteristic that the outer (or interior) solution, exact or asymptotic, generally cannot satisfy an admissible set of boundary conditions for the three-dimensional theory at a cylindrical edge of the plate. Near a cylindrical edge, the interior solution is supplemented by boundary layer solution components that becomes insignificant away from the edge. The boundary layer solution, even just a leading term approximation, needed to fit the edge data is usually very difficult to obtain.

Because of the difficulty associated with the residual boundary layer solution components, considerable effort has been made over the years to formulate the appropriate auxiliary conditions along the plate edge(s) for a two-dimensional linear thin or thick plate theory (without any reference to the boundary layer solution) that provides an interior solution for the plate problem accurate away from the plate edge(s) up to terms proportional to some power of the plate thickness. The only successful method for this purpose to date is based on a set of necessary (and sufficient) conditions for the exact edge data to induce a stress state that does not contain the interior solution [7-10]. Strictly speaking, these conditions for a decaying or residual (elastostatic) state have only been derived for the asymptotic limit; say when the plate thickness tends to zero with all other geometric and material parameters held fixed. Such asymptotic conditions provide the theoretical basis for the analysis of thin plate problems; they also suffice for moderately thick plate problems from a practical viewpoint. For thick plate theories, however, it is of fundamental interest to remove the restriction of asymptotic validity. The present paper shows how this can be done for important classes of boundary data associated with plane strain deformations or axisymmetric deformations. For these two classes of problems, the elimination of the asymptoticity restriction is made possible by a set of first integrals of the equations of elasticity established herein.

To allow for applications to a broader class of problems, the relevant first integrals will be derived for transversely isotropic materials. For added generality, we will need to obtain also the correct interior solutions for both plane strain and axisymmetric deformation of orthotropic plates analogous to the Levy solution $[12,17]$. Some applications of the results obtained in this paper will be discussed in Section 9; others can be found in references 15 and 16.

\section{Orthotropic plates in plane strain}

The behavior of an orthotropic plate under plane strain deformation is governed by the following sets of conditions:

(i) Two equilibrium equations for the three stress components $\boldsymbol{\sigma}_{x x}, \boldsymbol{\sigma}_{z z}$ and $\sigma_{x z}=\sigma_{z x}$ :

$$
\sigma_{x x, x}+\sigma_{z x, z}=0, \quad \sigma_{x z, x}+\sigma_{z z, z}=0
$$


(ii) Three stress strain relations relating the three strain components $\epsilon_{x x}, \epsilon_{z z}$ and $\epsilon_{x z}=\epsilon_{z x}$ to the three stres; components [1 ${ }^{1 ;}$

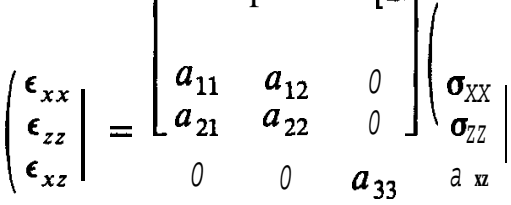

$$
\begin{aligned}
& =\left|\begin{array}{ccc}
\frac{1}{E_{1}} & -\nu_{12} & 0 \\
v_{21} & \frac{1}{\nu_{21}} & 0 \\
E_{1} & \frac{E_{2}}{2} & 1 \\
0 & 0 & \mathrm{G}
\end{array}\right|\left(\begin{array}{c}
\sigma_{x x} \\
\sigma_{z z} \\
\sigma_{x z}
\end{array}\right)
\end{aligned}
$$

with $a_{12}=a_{21}$ (and hence $\nu_{21} / E_{1}=\nu_{12} / E_{2}$ ).

(iii) Three strain-displacement relations defining the strain components in terms of the displacement components $\boldsymbol{u}_{x}$ and $\boldsymbol{u}_{\boldsymbol{z}}$ :

$$
\epsilon_{x x}=u_{x, x}, \quad \epsilon_{z z}=u_{z, z}, \quad \epsilon_{x z}=\epsilon_{z x}=u_{x y}+u_{z, x} .
$$

(iv) Appropriate boundary conditions on the surface of the plate.

In Equations (2.1)-(2.3), a comma indicates partial differentiation so that $0 \equiv \partial() / \partial t$. The elastic moduli $E_{j}, G$, and $\nu_{i j}$ in (2.2) are assumed to be constant for simplicity and $E_{j}$ and $G$ are necessarily positive. We have taken the equilibrium equations (2.1) to be homogeneous, as any distributed body load may be removed by a particular integral.

We are interested here in the plane strain problem governed by the differential Equations (2.1)-(2.3) for the plate strip $\{|z| \leq h,|x| \leq l,|y|<\infty\}$ with boundary conditions uniform in the $y$ direction. The top and bottom faces of the plate are taken to be traction free, so that

$$
z= \pm h: \sigma_{x z}=\sigma_{z z}=0
$$

The presence of any surface traction there may again be removed by a particular integral of the governing differential equations. The only forcing terms in the problem are prescribed along the edges $x= \pm 1(|z| \leq h,|y|<\infty)$ in terms of stress or displacement edge data in the form of one of the following four admissible combinations (along with $\boldsymbol{u}_{\boldsymbol{y}} \equiv 0$ ).

Case (1):

Case (2):

Case (3):

Case (4):

$$
\sigma_{x x}=\bar{\sigma}_{x x}(z), \quad \sigma_{x z}=\bar{\sigma}_{x z}(z)
$$


Evidently, we expect ( ), $\equiv 0, u_{y} \equiv \sigma_{x y} \equiv \sigma_{z y}=0$ in the elastostatic states induced by this data.

The two equilibrium equations (2.1) may be satisfied identically by expressing the stress components in terms of a stress function $\phi(x, z)$ :

$$
\sigma_{x x}=\phi_{, z z}, \quad \sigma_{z z}=\phi_{, x x}, \quad \sigma_{x z}=\sigma_{z x}=-\phi_{, x z} .
$$

The three strain components, defined in terms of only two displacement components by (2.3), satisfy the following differential equation of compatibility as in the isotropic case:

$$
\epsilon_{x z, x z}=\epsilon_{x x, z z}+\epsilon_{z z, x x}
$$

With the help of the stress strain relations (2.2), the strain components in Equation (2.10) can also be expressed in terms of $\phi$ so that we have the following equation for $\phi$ alone:

$$
a_{22} \phi_{, \mathrm{xxxx}}+\left(a_{12}+a_{21}+a_{33}\right) \phi_{, x x z z}+a_{11} \phi_{, \mathrm{zzzz}}=0
$$

or

$$
\frac{E_{1}}{E_{2}} \phi, x x x x+(2+\mu) \sqrt{\frac{E_{1}}{E_{2}}} \phi_{, x x z z}+\phi_{, z z z z}=0
$$

with

$$
\begin{gathered}
2+\mu=\frac{a_{12}+a_{21}+a_{33}}{\sqrt{a_{11} a_{22}}}=\stackrel{E}{G}-2 \nu \\
E=\sqrt{E_{1} E_{2}}, \quad \nu=\sqrt{\nu_{12} \nu_{21}} .
\end{gathered}
$$

For isotropic media, we have $\nu_{12}=\nu_{21}=\mathrm{v}$ and $E / G=2(1+\nu)$ so that $\mu=0$. In general, we have $\mu>-4$ as $\boldsymbol{\nu}$ is required to be no greater than unity by the requirement of positive definiteness of the strain energy of the plate (and hence the positive definiteness of the elastic coefficient matrix in Equation (2.2)).

\section{The exact interior and residual solution for plane strain deformations}

Let $\boldsymbol{\xi}=\boldsymbol{x} / \boldsymbol{l}$ and $\boldsymbol{\eta}=\boldsymbol{z} / \boldsymbol{h}$ and write (2.11b) as

$$
\epsilon^{4} \phi_{, \xi \xi \xi \xi}+(2+\mu) \epsilon^{2} \phi_{, \xi \xi \eta \eta}+\phi_{, \eta \eta \eta \eta}=0
$$

where $\epsilon=\left(E_{1} / E_{2}\right)^{1 / 4} h / l$. For $\epsilon \ll 1$, we designate as the interior (or outer) solution, $\boldsymbol{\phi}_{I}$, of our linear boundary value problem that portion of the exact solution (of the three-dimensional elasticity theory) that does not change signifi- 
cantly over a distance of the order of $l \epsilon$ in the $\mathrm{x}$-direction. To obtain this interior solution, we consider a perturbation solution for $\phi$ in the form,

$$
\phi_{I}(\xi, \eta ; \epsilon)=\sum_{k=0}^{\infty} \phi_{k}(\xi, \eta) \epsilon^{k}
$$

The coefficients $\boldsymbol{\phi}_{\boldsymbol{k}}, \boldsymbol{k}=\boldsymbol{0}, \boldsymbol{l}, 2, \ldots$, are required by Equation (3.1) to satisfy the following relations:

$$
\phi_{k, \eta \eta \eta \eta}=-(2+\mu) \phi_{k-2, \xi \xi \eta \eta}-\phi_{k-4, \xi \xi \xi \xi} \quad(k=0,1,2, \ldots)
$$

where quantities with a negative subscript are taken to be zero. Correspondingly, the boundary conditions on the plate faces require

$$
\eta= \pm 1: \quad \phi_{k, \xi \eta}=\phi_{k, \xi \xi}=0 \quad(k \geq 0)
$$

It follows from Equations (3.3)-(3.4) that we have

$$
\phi_{k}(\xi, \eta)=A_{k} \xi\left(\eta-\frac{1}{3} \eta^{3}\right)+B_{k} \eta^{2}+C_{k} \eta^{3} \quad(k \geq 0)
$$

where $\boldsymbol{A}_{\boldsymbol{k}}, \boldsymbol{B}_{\boldsymbol{k}}$ and $\boldsymbol{C}_{\boldsymbol{k}}$ are constants of integration.

With all coefficients in (3.2) having the same form (3.5), the series for $\phi$ may be summed to give,

$$
\phi_{I}=\frac{N_{0}}{4 h} z^{2}+\frac{M_{0}}{4 h^{3}} z^{3}-\frac{3 Q_{0}}{4 h} x\left(z-\frac{z^{3}}{3 h^{2}}\right),
$$

where

$$
\mathrm{Q}_{0}=-\frac{4}{3 l} \sum_{k=0}^{\infty} A_{k} \epsilon^{k}
$$

etc. It can now be verified by direct substitution that Equation (3.6) is an exact solution of (2.11) and (2.4), independent of the perturbation expansion (3.2) and the magnitude of $\boldsymbol{\epsilon}$. A more general expansion in powers of $\epsilon^{k / n}$ instead of (3.2) does not alter this exact solution. While the perturbation solution serves only as a vehicle to this exact solution and will not have any role in the subsequent development, the retention of a finite number of terms in the expansion (3.2) does correspond to a (thick) plate theory of a certain order. The physical meaning of the unknown constants $N_{0}, M_{0}$ and $Q_{0}$ may be seen from the following expressions for the stress components:

$$
\alpha^{\prime l x}=\frac{N_{0}}{2 h}+\frac{3 M_{0}}{2 h^{3}} z+\frac{3 Q_{0}}{2 h^{3}} x z, \quad 1_{x z}=\frac{3 Q_{0}}{4 h}\left(1-\frac{z^{2}}{h^{2}}\right), \quad \sigma_{z z}^{I}=0(3.7)
$$


with

$$
\int_{-h}^{h} \sigma_{x}^{I} d z=N_{0}, \quad \int_{-h}^{h} \sigma_{x z}^{I} d z=Q_{0}, \quad \int_{-h}^{h} \sigma_{x x}^{I} z d z=M_{0}+Q_{0} x
$$

The displacement components may be obtained from Equations (2.2), (2.3) and (3.7):

$$
\begin{aligned}
E_{1} u_{x}^{I}= & \frac{N_{0}}{2 h} x+\frac{3 M_{0}}{2 h^{3}} x z+\frac{Q_{0}}{4 h^{3}}\left(3 x^{2} z+\nu_{21} z^{3}-\frac{E_{1}}{G} z^{3}\right)+E_{1}[d-\omega z] \\
E_{1} u_{z}^{I}= & -\frac{\nu_{21} N_{0}}{2 h} z-\frac{3 M_{0}}{4 h^{3}}\left(\nu_{21} z^{2}+x^{2}\right)+\frac{Q_{0}}{4 h^{3}}\left(\frac{3 h^{2} E_{1}}{G} x-x^{3}-3 \nu_{21} x z^{2}\right) \\
& +E_{1}[\omega x+c] .
\end{aligned}
$$

Terms associated with the new constants of integration $\boldsymbol{\omega}, d$ and $\mathrm{c}$ in Equation (3.9) are of the nature of rigid body displacements.

The difference between the general exact solution of the plate problem and the interior solution (3.6) will be called the residual solution of the plate problem. Evidently, the residual solution does change significantly over a distance in the $\mathrm{x}$-direction of the order of $\boldsymbol{l} \epsilon$ when $\epsilon \ll 1$. In other words, we should expect the residual solution to change significantly as a function of $\zeta=\xi / \epsilon=x / l \epsilon$ over a dimensionless distance of order unity. In fact, we may write Equation (3.1) in terms of $\eta$ and $\xi$ :

$$
\phi_{, \zeta \zeta \zeta \zeta}+(2+\mu) \phi_{, \zeta \zeta \eta \eta}+\phi_{, \eta \eta \eta \eta}=0
$$

The fourth order Equation (3.10) admits product solutions of the form!

$$
\phi_{R}=e^{-\lambda \xi \hat{\Phi}}(\eta) \equiv e^{-\bar{\lambda} x} \Phi(z)
$$

where $\bar{\lambda}=\lambda / l \epsilon=A /(a h)$ with $a \equiv\left(E_{1} / E_{2}\right)^{1 / 4}$. For a residual solution, we must have $\lambda \neq 0$ as $\phi_{R}$ is expected to change significantly with $\zeta$.

With (3.11), Equation (3.10) becomes an ODE for $\hat{\boldsymbol{\Phi}}(\boldsymbol{\eta})$ :

$$
\lambda^{4} \hat{\Phi}+(2+\mu) \lambda^{2} \hat{\Phi}_{, \eta \eta}+\hat{\Phi}_{, \eta \eta \eta \eta}=0
$$

while the boundary conditions on the two faces become

$$
\eta= \pm 1: \quad \hat{\Phi}=\hat{\Phi}_{, \eta}=0
$$

\footnotetext{
${ }^{1}$ We will not be concerned with any matching of asymptotic expansions in this paper. Therefore, it will not be necessary to seek a perturbation solution of Equation (3.10). Such a solution does not lead to any simplification of the present problem.
} 
or

$$
\Phi( \pm h)=\Phi^{\prime}( \pm h)=0
$$

where ()$^{\prime} \equiv d() / d z$, keeping in mind that $\lambda \neq 0$ for the residual solution.

The homogeneous ordinary differential Equation (3.12) for $\hat{\boldsymbol{\Phi}}(\eta)$ and the four homogeneous boundary conditions in Equation (3.13) define an eigenvalue problem with $\lambda$ as the eigenvalue parameter. As in the isotropic case $(\mu=0)$, this problem has an infinite number of solutions $\left\{\lambda_{k}, \hat{\Phi}_{k}\right\}$ with $\lambda_{k}$ being a solution of

$$
\cos (\delta \lambda) \sin (\lambda / \delta)-\delta^{ \pm 2} \sin (\delta \lambda) \cos (\lambda / \delta)=0
$$

where

$$
\delta^{2}=1+\frac{1}{2} \mu+\sqrt{\left(1+\frac{1}{2} \mu\right)^{2}-1}
$$

Note that we have $\boldsymbol{\delta}^{2}>1$ for $\boldsymbol{\mu}>0$. The family of eigenvalues $\left\{\boldsymbol{\lambda}_{k}^{S}\right\}$ for the + sign in Equation (3.15) is associated with the eigenfunctions

$$
\hat{\Phi}_{S k}(\eta)=\cos \left(\lambda_{k}^{S} / \delta\right) \cos \left(\delta \lambda_{k}^{S} \eta\right)-\cos \left(\delta \lambda_{k}^{S}\right) \cos \left(\lambda_{k}^{S} \eta / \delta\right)
$$

which are even functions of $\eta$. Evidently, this family of solutions describes the extensional action of the plate. The other family $\left\{\lambda_{k}^{B}\right\}$ for the - sign in Equation (3.15) has as its family of eigenfunctions,

$$
\hat{\Phi}_{B k}(\eta)=\sin \left(\lambda_{k}^{B} / \delta\right) \sin \left(\delta \lambda_{k}^{B} \eta\right)-\sin \left(\delta \lambda_{k}^{B}\right) \sin \left(\lambda_{k}^{B} \eta / \delta\right)
$$

which are odd functions of $\eta$. This family is for the plate bending (and flexure) portion of the problem.

As in the isotropic case $[5,61$, we expect

$$
\phi=\phi_{I}+\phi_{R}
$$

where $\phi_{I}$ is the interior solution obtained earlier and

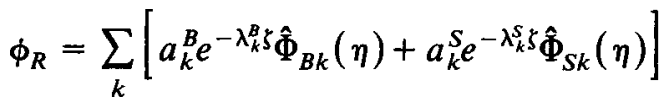

$$
\begin{aligned}
& =\sum_{k}\left[a_{k}^{B} e^{-\bar{\lambda}_{k}^{B} x} \Phi_{B k}(z)+a_{k}^{S} e^{-\bar{\lambda}_{k}^{S} x} \Phi_{S k}(z)\right]
\end{aligned}
$$

with $\bar{\lambda}_{k}=\lambda_{k} /(a h)=\lambda_{k} /(l \boldsymbol{\epsilon})$ as previously introduced in Equation (3.11). Note that $\phi_{R}$ is also an exact solution of (2.11) and $\phi_{I}$ in fact consists of the eigenfunctions of (3.12)-(3.13) with $\lambda=0$. The coefficients $\left\{a_{k}^{B}\right\},\left\{a_{k}^{S}\right\}, N_{0}, M_{0}$ and $Q_{0}$ (in $\phi_{I}$ ) are to be determined by the edge data at $x= \pm l$. As the solutions 
for $\left\{\boldsymbol{\lambda}_{\boldsymbol{k}}\right\}$ and $\left\{\boldsymbol{a}_{\boldsymbol{k}}\right\}$ are generally tedious and costly, there is considerable interest in obtaining $N_{0}, Q_{0}$ and $M_{0}$ without an explicit (or numerical) solution for the residual solution. This will be accomplished by obtaining an appropriate set of auxiliary conditions along the plate edge for the interior solution alone.

The technique developed in references 7-10 for formulating the proper edge conditions for correct isotropic plate theory solutions depends critically on a set of integral relations which must be satisfied by the edge data in order for the data to induce only a residual solution. By way of the Betti-Rayleigh reciprocal theorem, the needed necessary conditions have been derived for isotropic plates valid asymptotically as $h \rightarrow 0$. While they suffice for thin plate problems adequately approximated by the Germain-Kirchhoff theory; it is desirable to have a similar set of conditions without the asymptoticity restriction for thick plate problems. We will obtain these conditions in the Section (5) for the more general orthotropic plate case with stress or mixed edge data listed as Cases (1)-(3) in Section 2. These new exact necessary conditions for a residual state are direct consequences of the first integrals of the equations of elasticity derived in the next section.

\section{Six first integrals for plane strain deformations}

In this section, we derive six first integrals from the governing differential equations for plane strain deformations and the traction-free conditions on the two faces of the plate. Three of the six are relatively straightforward. Upon integrating (2.1) across the plate thickness and using the boundary conditions (2.4) [or (3.14)], we get;

$$
\left[\int_{-h}^{h} \sigma_{x x} d z\right]_{, x}=0, \quad\left[\int_{-h}^{h} \sigma_{x z} d z\right]_{, x}=0
$$

or

$$
\int_{-h}^{h} \sigma_{x x} d z=C_{1}, \quad \int_{-h}^{h} \sigma_{x z} d z=C_{2}
$$

A third integrated relation may be obtained by multiplying the first equation of (2.1) by $\mathrm{z}$ and integrating with respect to $\mathrm{z}$ to get

$$
\left[\int_{-h}^{h} \sigma_{x x} z d z\right]_{, x x}=\left[\left.z \sigma_{x z}\right|_{-h} ^{h}+\int_{-h}^{h} \sigma_{x z} d z\right]_{, x}=\left[\sigma_{z z}\right]_{-h}^{h}=0
$$

so that

$$
\int_{-h}^{\mathrm{h}} \sigma_{x x} z d z=C_{3} x+C_{4}
$$


On the other hand, we have from Equations (3.8a) and (3.19b)

$$
\begin{aligned}
\int_{-h}^{h} \sigma_{x x} d z & =\int_{-h}^{h}\left[\sigma_{x x}^{I}+\sigma_{\lambda}{ }_{k}^{k} d z=N_{0}+\int_{-h}^{h} \sum_{k} a_{k}^{S} e^{-\bar{\lambda}_{k}^{S} x} \Phi_{S k}^{\prime \prime}(z) d z\right. \\
& =N_{0}+\sum a_{k}^{S} e^{-\bar{\lambda}_{k}^{S} x}\left[\Phi_{S k}^{\prime}(z)\right]_{-h}^{h}=N_{0}
\end{aligned}
$$

to get $C_{1}=N_{0}$. Similar considerations give $C_{2}=Q_{0}$ and $C_{3}=M_{0}$. Thus, the three first integrals (4.2) and (4.3) may be taken to be

$$
\begin{aligned}
& \int_{-h}^{h} \sigma_{x x} d z=N_{0}, \\
& \int_{-h}^{h} \sigma_{x z} d z=Q_{0}, \\
& \int_{-h}^{h} \sigma_{x x} z d z=Q_{0} x+M_{0} .
\end{aligned}
$$

They may also be deduced by the usual requirement of global equilibrium.

The other three first integrals are not at all obvious and cannot be deduced from overall equilibrium considerations alone. They were in fact suggested by the relevant asymptotic necessary conditions for a residual state for isotropic plates obtained in [7]. We begin the derivation by integrating $\boldsymbol{\epsilon}_{\boldsymbol{x} x}$ in Equations (2.2) and (2.3) across the plate thickness to get,

$$
\left[\int_{-h}^{h} u_{x} d z\right]_{, x}=a_{11} \int_{-h}^{h} \sigma_{x x} d z+a_{12} \int_{-h}^{h} \sigma_{z z} d z
$$

Now, we observe the relation

$$
\int_{-h}^{h} \sigma_{z z} d z=\left[z \sigma_{z z}\right]_{-h}^{h}-\int_{-h}^{h} z \sigma_{z z, z} d z=\left[\int_{-h}^{h} z \sigma_{x z} d z\right]_{, x}
$$

so that (4.6) may be written in the form of a fourth integral

$$
\int_{-h}^{h}\left(u_{x}-a_{12} z \sigma_{x z}\right) d z=a_{11} N_{0} x+C_{4} \text {. }
$$

We may also multiply $\boldsymbol{\epsilon}_{\boldsymbol{x} \boldsymbol{x}}$ in Equations (2.2) and (2.3) byz and integrate to get

$$
\begin{aligned}
{\left[\int_{-h}^{h} u_{x} z d z\right]_{, x} } & =a_{11}\left(Q_{0} x+M_{0}\right)+a_{12} \int_{-h}^{h} \sigma_{z z} z d z \\
& =a_{11}\left(Q_{0} x+M_{0}\right)+a_{12}\left[\int_{-h}^{h} 1 / 2 z^{2} \sigma_{x z} d z\right], x
\end{aligned}
$$


The fifth integral follows from Equation (4.9) in the form

(v) $\quad \int_{-h}^{h}\left[z u_{x}-1 / 2 a_{12} z^{2} \sigma_{x z}\right] d z=a_{11}\left(1 / 2 Q_{0} x^{2}+M_{0} x\right)+C_{5}$.

For the sixth integral, we note the following two relations which follow from the expression for $\epsilon_{x z}$ in Equations (2.2) and (2.3):

$$
\begin{aligned}
a_{33} \int_{-h}^{h} \sigma_{x z} d z & =\int_{-h}^{h}\left(u_{z, x}+u_{x, z}\right) d z=\left[\int_{-h}^{h} u_{z} d z\right]_{, x}+\left[u_{x}\right]_{-h}^{h} \\
a_{33} \int_{-h}^{h} \sigma_{x z} z^{2} d z & =\left[\int_{-h}^{h} u_{z} z^{2} d z\right]_{, x}+\left[z^{2} u_{x}\right]_{-h}^{h}-2 \int_{-h}^{h} u_{x} z d z
\end{aligned}
$$

The relations (4.11) and (4.10) can be used to eliminate the second and third term on the right side of (4.12) to get

$$
\begin{aligned}
{\left[\int_{-h}^{h}\left(h^{2}-z^{2}\right) u_{z} d z\right]_{, x}=} & -2 \int_{-h}^{h} u_{x} z d z+a_{33} \int_{-h}^{h}\left(h^{2}-z^{2}\right) \sigma_{x z} d z \\
= & a_{33} h^{2} Q_{0}-a_{11}\left(Q_{0} x^{2}+2 M_{0} x\right)-2 C_{5} \\
& -\left(a_{12}+a_{33}\right) \int_{-h}^{h} z^{2} \sigma_{x z} d z
\end{aligned}
$$

With

$$
\int_{-h}^{h} z^{2} \sigma_{x z} d z=\left[1 / 3 z^{3} \sigma_{x z}\right]_{-h}^{h}+\left[\int_{-h}^{h} 1 / 3 z^{3} \sigma_{x x} d z\right]_{, x}
$$

the relation (4.13) may be written as the following sixth integral:

$$
\begin{aligned}
\int_{-h}^{h} & {\left[\left(h^{2}-z^{2}\right) u_{z}+1 / 3\left(a_{12}+a_{33}\right) z^{3} \sigma_{x x}\right] d z } \\
& =C_{6}+\left(a_{33} h^{2} Q_{0}-2 C_{5}\right) x-a_{11}\left(1 / 3 Q_{0} x^{3}+M_{0} x^{2}\right) .
\end{aligned}
$$

The integrals (i)-( vi) will be used to determine necessary conditions for a plane strain state to be a residual state in the next section.

\section{Necessary conditions for a residual state in plane strain deformations}

For stress or mixed data at an edge $\mathrm{x}=L(= \pm l)$, the relevant necessary conditions for the absence of the outer solution in the induced plane strain deformations can now be deduced from the results of the last section. In general, we must have $N_{0}=Q_{0}=M_{0}=0$ for an elastic state to be a residual state. So, the 
right-hand side of the first integrals ( $i)$-(iii) in Equation (4.5) vanishes for any station $x$. By setting $x=L$, these first integrals require the stress data

$$
\sigma_{x x}=\bar{\sigma}_{x x}(z), \quad \sigma_{x z}=\bar{\sigma}_{x z}(z),
$$

of Case (1) to give rise to no resultant force and bending moment. We have therefore the following result for stress edge data:

Case (1). For the stress data to induce only a residual state, the edge tractions $\overline{\boldsymbol{\sigma}}_{x x}(z)$ and $\overline{\boldsymbol{\sigma}}_{x z}(z)$ must satisfy the following three (necessary) conditions:

$$
\int_{-h}^{h} \bar{\sigma}_{x x} d z=0, \quad \int_{-h}^{h} \bar{\sigma}_{x z} d z=0, \quad \int_{-h}^{h} \bar{\sigma}_{x x} z d z=0
$$

The result is similar to that obtained in [6] but now the theorem holds without an error term on the right side of the three equations in (5.1) of the form $0\left(e^{-\gamma l / h}\right)$ that is exponentially small only for $h \ll \gamma l$.

For the mixed data in Case (2), it is necessary again to have $N_{0}=Q_{0}=M_{0}=0$ for a residual state $\left\{\mathbf{u}^{R}, \boldsymbol{\sigma}^{R}\right\}$. The second integral then requires that $\overline{\boldsymbol{\sigma}}_{x z}(\mathrm{z})$ gives rise to no resultant transverse force while the fourth and fifth integral relations (4.8) and (4.10) reduce to

$$
\int_{-h}^{h}\left[u_{x}^{R}-a_{12} z \sigma_{x z}^{R}\right] d z=C_{4}, \quad \int_{-h}^{h}\left[z u_{x}^{R}-1 / 2 a_{12} z^{2} \sigma_{x x}^{R}\right] d z=C_{5} .
$$

For a residual state, the constants $C_{4}$ and $C_{5}$ must also vanish. To get $C_{4}=0$, we note:

$$
\begin{aligned}
u_{x, x}^{R} & =a_{11} \sigma_{x x}^{R}+a_{12} \sigma_{z z}^{R} \\
& =\sum_{k}\left\{a_{k}^{B} e^{-\bar{\lambda}_{k}^{B} x}\left[a_{11} \Phi_{B k}^{\prime \prime}+a_{12}\left(\bar{\lambda}_{k}^{B}\right)^{2} \Phi_{B k}\right]+a_{k}^{S} e^{-\bar{\lambda}_{k}^{S} x}\left[a_{11} \Phi_{S k}^{\prime \prime}+a_{12}\left(\bar{\lambda}_{k}^{S}\right)^{2} \Phi_{S k}\right]\right\}
\end{aligned}
$$

or

$$
u_{x}^{R}=-\sum_{k}\left\{a_{k}^{B} e^{-\bar{\lambda}_{k}^{B} x}\left[\frac{a_{11}}{\bar{\lambda}_{k}^{B}} \Phi_{B k}^{\prime \prime}+a_{12} \bar{\lambda}_{k}^{B} \Phi_{B k}\right]+a_{k}^{S} e^{-\bar{\lambda}_{k}^{S} x}\left[\frac{a_{11}}{\bar{\lambda}_{k}^{S}} \Phi_{S k}^{\prime \prime}+a_{12} \bar{\lambda}_{k}^{S} \Phi_{S k}\right]\right\}
$$

(as the constant of integration must vanish for a residual displacement field) so that

$$
\begin{aligned}
\int_{-h}^{h}\left(u_{x}^{R}-a_{12} z \sigma_{x z}^{R}\right) d z & =-\sum_{k} a_{k}^{s} e^{-\bar{\lambda}_{k}^{s} x} \int_{-h}^{h}\left[\frac{a_{11}}{\bar{\lambda}_{k}^{s}} \Phi_{S k}^{\prime \prime}+a_{12} \bar{\lambda}_{k}^{s} \Phi_{S k}+a_{12} \bar{\lambda}_{k}^{S} z \Phi_{S k}^{\prime}\right] d z \\
& =-\sum_{k} a_{k}^{S} e^{-\bar{\lambda}_{k}^{s} x}\left[\frac{a_{11}}{\bar{\lambda}_{k}^{s}} \Phi_{S k}^{\prime}+a_{12} \bar{\lambda}_{k}^{s} z \Phi_{S k}\right]_{-h}^{h}=0
\end{aligned}
$$


To get $\boldsymbol{C}_{5}=0$, we consider the following combination of $\boldsymbol{u}_{\boldsymbol{x}}^{\boldsymbol{R}}$ and $\boldsymbol{\sigma}_{\boldsymbol{x} z}^{\boldsymbol{R}}$ :

$$
z u_{x}^{R}-1 / 2 a_{12} z^{2} \sigma_{x z}^{R}=\int^{x}\left(a_{11} \sigma_{x x}^{R}+a_{12} \sigma_{z z}^{R}\right) z d x-1 / 2 a_{12} z^{2} \sigma_{x z}^{R}
$$

which can be expressed in terms of the eigenfunctions $\left\{\Phi_{k}\right\}$ with the help of the results for residual states in Section 3 . We integrate the resulting expression across the thickness to get

$$
\begin{aligned}
\int_{-h}^{h}\left(z u_{x}^{R}-\frac{1}{2} a_{12} z^{2} \sigma_{x z}^{R}\right) d z \\
\quad=-\sum_{k} a_{k}^{B} e^{-\bar{\lambda}_{k}^{B} x}\left[\frac{a_{11}}{\bar{\lambda}_{k}^{B}}\left(z \Phi_{B k}^{\prime}-\Phi_{B k}\right)-1 / 2 a_{12} \bar{\lambda}_{k}^{B} z^{2} \Phi_{B k}\right]_{-h}^{h}=0
\end{aligned}
$$

for any station $|x| \leq l$. In particular, we get by setting $x=L(= \pm l)$ the following result analogous to the asymptotic results for Case (2) obtained in reference [7], but now without the asymptoticity (and isotropy) restriction:

Case 2. For the mixed data (2.6) to induce only a residual elastostatic state, the data must satisfy the three conditions

$$
\begin{aligned}
\int_{-h}^{h} \bar{\sigma}_{x z} d z & =0, \\
\int_{-h}^{h}\left(\bar{u}_{x}-a_{12} z \bar{\sigma}_{x z}\right) d z & =0, \\
\int_{-h}^{h}\left(z \bar{u}_{x}-1 / 2 a_{12} z^{2} \bar{\sigma}_{x z}\right) d z & =0
\end{aligned}
$$

As an illustration of the use of the above result, consider an infinitely long strip of thickness $2 h$ and width $2 l$. The two faces $z= \pm h$ are free of tractions. The two edges $x= \pm 1$ are free of transverse shear stress so that we have $\sigma_{x z} \equiv 0$ there; they are uniformly stretched in the x-direction resulting in an edge displacement of $\boldsymbol{u}_{x}= \pm u_{0}$. The residual solutions $\tilde{\sigma}_{x z} \equiv \sigma_{x z}-\sigma_{x z}^{I}$ and $\tilde{u}_{x} \equiv u_{x}-u_{x}^{I}$ of the resulting state of plane strain must satisfy (5.8) at the two edges. With $\sigma_{x z}=0$ at $x= \pm l$, the condition (5.8a) requires $Q_{0}=0$ while the conditions (5.8b) and (5.8c) become (see (3.7) and (3.9)) $\pm N_{0} l+2 h d= \pm 2 h u_{0}$ and $\pm M_{0} l-$ $2 E_{1} h^{3} \omega / 3=0$. These four relations give $d=\omega=M_{0}=0$ and $N_{0}=2 h u_{0}$. The interior solution for the problem is thereby determined up to a rigid translation in the z-direction.

For the mixed data in Case (3), we have from the requirements $N_{0}=Q_{0}=M_{0}$ $=0$ for a residual state that the edge traction $\overline{\boldsymbol{\sigma}}_{\boldsymbol{x}}(\mathrm{z})$ should give rise to no resultant force and bending moment (by overall equilibrium requirements (4.5)), while the first integral (vi) reduces to

$$
\int_{-h}^{h}\left[\left(h^{2}-z^{2}\right) u_{z}^{R}+\frac{1}{3}\left(a_{12}+a_{33}\right) z^{3} \sigma_{x x}^{R}\right] d z=C_{6}
$$


where we have made use of a previous result from Equation (5.7) to set $C_{5}=0$. For a residual state, we must have $C_{6}=0$ also by an argument similar to the one above that gives $C_{4}=0$. The relation (5.9) with $C_{6}=0$ holds for all station $|x| \leq l$. By taking $x=L(= \pm l)$, we get for the mixed data of Case (3) the following result:

Case (3). If the mixed data (2.7) gives rise only to a residual state, then the data must satisfy the following three relations

$$
\begin{gathered}
\int_{-h}^{h} \overline{\boldsymbol{\sigma}}_{x x} d z=0, \\
\int_{-h}^{h} \overline{\boldsymbol{\sigma}}_{x x} z d z=0, \\
\int_{-h}^{h}\left[\left(h^{2}-z^{2}\right) \bar{u}_{z}+\frac{1}{3}\left(a_{33}+a_{12}\right) \bar{\sigma}_{x x} z^{3}\right] d z=0 .
\end{gathered}
$$

The result is similar to the corresponding theorem obtained in [7] by the reciprocal theorem for the isotropic case, but now without the asymptoticity (and isotropy) restriction.

For a simple application, we consider the same infinitely long strip treated in Case 2. If the edge conditions at $x= \pm 1$ are replaced by $\sigma_{x x} \equiv 0$ and $u_{z}= \pm w_{0}$ instead, then $(5.10 \mathrm{a}, \mathrm{b})$ applied to the residual solution at both edges require $N_{0}=Q_{0}=M_{0}=0$. The remaining condition (5.10c) requires that $\mathrm{c}=0$ and $\boldsymbol{\omega}=$ $w_{0} / l$ so that the interior solution is just a rigid body motion. With both edge conditions satisfied exactly by the interior solution, the residual solution must satisfy the homogenous edge conditions $\tilde{\boldsymbol{\sigma}}_{\boldsymbol{x} x}=0$ and $\tilde{\boldsymbol{u}}_{z}=0$ at $\boldsymbol{x}= \pm 1$ and must therefore vanish identically for this problem. Thus the exact solution of the problem is just the interior solution consisting of a rigid rotation possibly with a rigid translation in the $\mathrm{x}$-direction.

We have not been able to remove the asymptoticity restriction from the result obtained in [7] for displacement data. This lack of success may be related to the fact that no elementary solution for the canonical problems needed for the application of the reciprocal theorem could be found for that case. A more thorough discussion for this case can be found in $[15,16]$.

Other applications of the results obtained in this section will be discussed later in Section 9.

\section{Axisymmetric deformation of transversely isotropic plates}

The two homogeneous equations of equilibrium for the four relevant stress components, $\sigma_{r r}, \boldsymbol{\sigma}_{\theta \theta}, \boldsymbol{\sigma}_{z z}$ and $\boldsymbol{\sigma}_{r z}=\sigma_{z r}$, in elastostatic problems with axisymmetry may be taken in the form:

$$
\begin{array}{r}
\left(r \sigma_{r r}\right)_{, r}-\sigma_{\theta \theta}+r \sigma_{r z, z}=0, \\
\left(r \sigma_{r z}\right)_{, r}+r \sigma_{z z, z}=0 .
\end{array}
$$


The relevant strain measures, $\boldsymbol{\epsilon}_{r \boldsymbol{r}}, \boldsymbol{\epsilon}_{\boldsymbol{\theta} \boldsymbol{\theta}}, \boldsymbol{\epsilon}_{z z}$ and $\boldsymbol{\epsilon}_{\boldsymbol{r} z}=\boldsymbol{\epsilon}_{z \boldsymbol{r}}$, are related to the stress components by

$$
\begin{aligned}
& \epsilon_{r r}=a_{11} \sigma_{r r}+a_{12} \sigma_{\theta \theta}+a_{13} \sigma_{z z}, \\
& \epsilon_{\theta \theta}=a_{21} \sigma_{r r}+a_{22} \sigma_{\theta \theta}+a_{23} \sigma_{z z} \\
& \epsilon_{z z}=a_{31} \sigma_{r r}+\sigma_{32} \sigma_{\theta \theta}+a_{33} \sigma_{z z}, \\
& \epsilon_{r z}=a_{44} \sigma_{r z} .
\end{aligned}
$$

For simplicity, we limit our analysis to transversely isotropic materials with $a_{22}=a_{11}, a_{21}=a_{12}$ and $a_{32}=a_{23}=a_{31}=a_{13}$ where

$$
\begin{aligned}
& a_{11}=\frac{1}{E}, \quad a_{12}=-\frac{v}{E}, \quad a_{33}=\frac{1}{E_{3}}, \\
& a_{13}=-\frac{\nu_{3}}{E}=-\frac{\nu}{E_{3}}, \quad a_{44}=\frac{1}{G} .
\end{aligned}
$$

The strain components are defined in terms of the radial and axial displacement components $\boldsymbol{u}_{r}$ and $\boldsymbol{u}_{\boldsymbol{z}}$ by

$$
\begin{aligned}
& \epsilon_{r r}=U_{r, r}, \quad \epsilon_{\theta \theta}=\frac{1}{r} u_{r}, \quad \epsilon_{z z}=U_{z, z}, \\
& \epsilon_{r z}=\epsilon_{z r}=U_{r, z}+u_{z, r} .
\end{aligned}
$$

It is not difficult to verify that these strain measures satisfy the following two compatibility equations:

$$
\left(r \epsilon_{\theta \theta}\right)_{, r}=\epsilon_{r r}, \quad \epsilon_{r r, z z}+\epsilon_{z z, r r}=\epsilon_{r z, r z} .
$$

Again, we consider a flat body which extends from $z=-\boldsymbol{h}$ to $z=h$ in the transverse direction with the two faces free of surface traction, so that

$$
z= \pm h: \sigma_{r z}=\sigma_{z z}=0 .
$$

In Section 7, we will discuss specific admissible combinations of stress and displacement conditions along the cylindrical edge(s) to complete the problem formulation.

As in the isotropic case, the equilibrium and compatibility equations are satisfied identically by the stress function representation [13]

$$
\begin{aligned}
& \sigma_{r r}=-\frac{\partial}{\partial z}\left[\frac{\partial^{2}}{\partial r^{2}}+\frac{\alpha_{2}}{r} \frac{\partial}{\partial r}+\alpha_{1} \frac{\partial^{2}}{\partial z^{2}}\right] \phi, \quad \sigma_{z z}=\frac{\partial}{\partial z}\left[\alpha_{3} \Delta_{r}+\alpha_{4} \frac{\partial^{2}}{\partial z^{2}}\right] \phi \\
& \sigma_{\theta \theta}=-\frac{\partial}{\partial z}\left[\alpha_{2} \frac{\partial^{2}}{\partial r^{2}}+\frac{1}{r} \frac{\partial}{\partial r}+\alpha_{1} \frac{\partial^{2}}{\partial z^{2}}\right] \phi \\
& \sigma_{r z}=\sigma_{z r}=\frac{\partial}{\partial r}\left[\Delta_{r}+\alpha_{1} \frac{\partial^{2}}{\partial z^{2}}\right] \phi
\end{aligned}
$$


with

$$
\Delta_{r}() \equiv()_{, r r}+r^{-1}()_{, r}
$$

and

$$
\begin{aligned}
& \beta \alpha_{1}=a_{13}\left(a_{11}-a_{12}\right), \quad \beta \alpha_{2}=a_{13}\left(a_{13}+a_{44}\right)-a_{12} a_{33} \\
& \beta \alpha_{3}=a_{13}\left(a_{11}-a_{12}\right)+a_{11} a_{44}, \quad \beta \alpha_{4}=a_{11}^{2}-a_{12}^{2}
\end{aligned}
$$

where $\beta=a_{11} a_{33}-a_{13}^{2}$ and $\phi$ satisfies the fourth order partial differential equation

$$
\begin{gathered}
{\left[\Delta_{r}^{2}+\left(\alpha_{1}+\alpha_{3}\right) \Delta_{r} \frac{\partial^{2}}{\partial z^{2}}+\alpha_{4} \frac{\partial^{4}}{\partial z^{4}}\right] \phi \equiv\left[\Delta_{r}+\frac{1}{s_{1}^{2}} \frac{\partial^{2}}{\partial z^{2}}\right]\left[\Delta_{r}+\frac{1}{s_{2}^{2}} \frac{\partial^{2}}{\partial z^{2}}\right] \phi=0} \\
\left\{s_{1}^{2}, s_{2}^{2}\right\}=\frac{1}{2 \alpha_{4}}\left\{\left(\alpha_{1}+\alpha_{3}\right) \pm \sqrt{\left(\alpha_{1}+\alpha_{3}\right)^{2}-4 \alpha_{4}}\right\}
\end{gathered}
$$

For isotropic materials, we have $2 \alpha_{4}=\alpha_{1}+\alpha_{3}=2$ and therewith $s_{1}=s_{2}=1$ so that Equation (6.9) reduces correctly to a biharmonic equation with axisymmetry. With $\alpha_{1}=\alpha_{2}=-\nu /(1-\nu)$ and $\alpha_{3}=(2-\nu) /(1-\nu)$, the representation (6.7) also reduces correctly to the corresponding representation for the isotropic case (cf. (5.2) in [8]). For our more general orthotropic plate, the positive definiteness of the strain energy of the plate requires $\boldsymbol{\beta}>0$ and $\boldsymbol{\alpha}_{\mathbf{4}}>0$.

Let

$$
x=r / r_{0}, \quad \mathrm{y}=z / h, \quad \epsilon^{2}=h^{2} / r_{0}^{2},
$$

and write Equation (6.9) as

$$
\left(\epsilon^{2} D^{2}+\frac{1}{s_{2}^{2}} \frac{\partial^{2}}{\partial y^{2}}\right)\left(\epsilon^{2} D^{2}+\underset{s_{1}^{2}}{1} \frac{\partial 2}{\partial y^{2}}\right) \phi=0
$$

when $D^{2}() \equiv()_{, x x}+x^{-1}()_{, x}$. The two boundary conditions in Equation (6.6) may also be written in terms of $\phi, x, y$ and $\epsilon$ :

$$
Y= \pm 1: \quad\left\{\begin{array}{l}
\frac{\partial}{\partial x}\left[\epsilon^{2} D^{2}+\alpha_{1} \frac{\partial^{2}}{\partial y^{2}}\right] \phi=0 \\
\frac{\partial}{\partial y}\left[\alpha_{3} \epsilon^{2} D^{2}+\alpha_{4} \frac{\partial^{2}}{\partial y^{2}}\right] \phi=0 .
\end{array}\right.
$$


For thin plates with $\epsilon \ll 1$, we consider a perturbation solution for $\phi$ in the form,

$$
\phi_{I}=\sum_{k=0}^{\infty} \phi_{k}(x, y) \epsilon^{k}
$$

The differential Equation (6.12) and the boundary conditions (6.13) require the coefficients $\left\{\boldsymbol{\phi}_{k}\right\}$ to be determined by the following sequence of boundary value problems:

$$
\begin{gathered}
\phi_{k, y y y y}+\left(s_{1}^{2}+s_{2}^{2}\right) D^{2} \phi_{k-2, y y}+s_{1}^{2} s_{2}^{2} D^{4} \phi_{k-4}=0 \\
y= \pm 1: \quad\left\{\begin{array}{l}
{\left[\alpha_{1} \phi_{k, y y}+D^{2} \phi_{k-2}\right]_{, x}=0} \\
{\left[\alpha_{4} \phi_{k, y y}+\alpha_{3} D^{2} \phi_{k-2}\right]_{, y}=0}
\end{array}\right.
\end{gathered}
$$

where $\boldsymbol{k}=0,1,2,3 \ldots$ and terms with a negative subscript vanish identically. It is straightforward to obtain the exact solutions of these simple BVPs in terms of elementary functions; the detailed calculations can be found in the Appendix. As in the plane strain case, the resulting perturbation solution (6.14) can be summed to give the following expression for $\phi_{I}$ :

$$
\phi_{I}=a \phi_{a}+b \phi_{b}+c \phi_{c}+d \phi_{d}+e \phi_{e}
$$

with

$$
\begin{aligned}
\phi_{a}= & -\frac{2}{\alpha_{1}} z^{2}[\ln (r)+1]+r^{2} \ln (r), \\
\phi_{b}= & \frac{8 \alpha_{3}}{3 \alpha_{1} \alpha_{4}} z^{4}-\frac{8}{\alpha_{1}} z^{2} r^{2}+r^{4}, \\
\phi_{c}= & \frac{4 \alpha_{3}}{3 \alpha_{1} \alpha_{4}} z^{4}[2 \ln (r)+3]-\frac{4}{\alpha_{1}} z^{2} r^{2}[2 \ln (r)+1] \\
& +\frac{8\left(\alpha_{4}-\alpha_{1} \alpha_{3}\right)}{\alpha_{1}^{2} \alpha_{4}} z^{2} h^{2}[2 \ln (r)+3]+r^{4} \ln (r), \\
\phi_{d}= & \frac{z}{h} \ln (r), \\
\phi_{e}= & -\frac{\alpha_{3} z^{3}}{6 \alpha_{4} h}+\frac{r^{2} z}{4 h} .
\end{aligned}
$$


Correspondingly, we have:

$$
\begin{aligned}
& \sigma_{r z}^{I}=\frac{c}{r}\left(\alpha_{4}-\alpha_{1} \alpha_{3}\right)\left(h^{2}-z^{2}\right) \\
& \sigma_{r r}^{I}=a \frac{z}{r^{2}}+b z+c\left[\alpha_{3}\left(1-\alpha_{2}\right) \frac{z^{3}}{3 r^{2}}+\left\{\alpha_{4}\left(1+\alpha_{2}\right)-2 \alpha_{1} \alpha_{3}\right\} z \ln (r)+\frac{d}{r^{2}}+\right. \\
& \begin{array}{r}
\sigma_{\theta \theta}^{I}=-a \frac{z}{r^{2}}+b z+c\left[\alpha_{3}\left(\alpha_{2}-1\right) \frac{z^{3}}{3 r^{2}}+\left\{\alpha_{4}\left(1+\alpha_{2}\right)-2 \alpha_{1} \alpha_{3}\right\} z \ln (r)\right. \\
+\alpha_{4}\left(\alpha_{2}-1\right) z-\frac{d}{r^{2}}+\mathrm{e}
\end{array}
\end{aligned}
$$

Expressions for other stress and displacement components are not listed here (but can be found in [14]) as they will not be needed in the subsequent development.

The expression (6.18) for $\boldsymbol{\phi}_{I}$ may be grouped differently; e.g., in powers of $z$. However, it is evident that those terms in even powers of $\mathrm{z}$ are associated with the bending action and the remaining terms with stretching. We wish to emphasize that the interior solution (6.18), though obtained by way of an outer asymptotic expansion procedure, is an exact solution of (6.12) and (6.13) independent of the magnitude of the parameter $\boldsymbol{\epsilon}$. It is equivalent to the Levy solution for the isotropic case and reduces to that solution upon setting $a_{11}=a_{33}=1 / E$, $a_{12}=a_{13}=-\nu / E$ and $a_{44}=2(1+\nu) / E$.

The interior solution (6.18) does not change significantly over a distance of the order of the plate thickness (or more precisely of the order of $\epsilon \boldsymbol{r}_{0}$ ) in the radial direction. For $\epsilon \ll 1$, we again consider supplementary residual solutions which do change significantly over a distance of order $\epsilon r_{0}$. These solutions may be treated as smoothly varying functions of $t=x / \epsilon$ and Y. We therefore set $D_{t}^{2}() \bar{\equiv}()_{, t t}+t^{-1}()_{, t}$ and write Equations (6.12) and (6.13) as

$$
\left(D_{t}^{2}+\frac{1}{s_{2}^{2}} \frac{\partial^{2}}{\partial y^{2}}\right)\left(D_{t}^{2}+\frac{1}{s_{1}^{2}} \frac{\partial^{2}}{\partial y^{2}}\right) \phi=0
$$

and

$$
\begin{aligned}
& y= \pm 1: \frac{\partial}{\partial t}\left(D_{t}^{2}+\alpha_{1} \frac{\partial^{2}}{\partial y^{2}}\right) \phi=0 \\
& \frac{\partial}{\partial y}\left(\alpha_{3} D_{t}^{2}+\alpha_{4} \frac{\partial^{2}}{\partial y^{2}}\right) \phi=0,
\end{aligned}
$$

respectively. It is evident from Equations (6.12') and (6.13') that when treated as 
a function of $\boldsymbol{t}$ and $\boldsymbol{y}, \boldsymbol{\phi}$ does not depend on the parameter $\epsilon$ explicitly, and an expansion of $\boldsymbol{\phi}$ in powers of $\boldsymbol{\epsilon}$ does not lead to any simplification of the problem. As we will work directly with (6.12'), which is equivalent to (6.9) and (6.12), the supplementary residual solution obtained will also be an exact solution of the plate problem.

Analogous to the plane strain case, Equations (6.12') and (6.13') admit product solutions of the form

$$
\phi_{R}=I_{0}(\lambda t) \hat{\Phi}(y) \equiv I_{0}(\bar{\lambda} r) \Phi(z)
$$

where $\bar{\lambda}=\mathrm{A} /\left(r_{0} \epsilon\right)$ and $I_{0}(\mathrm{I})$ is the modified Bessel function of order zero of the first kind. (A Bessel function of the second kind should also be considered for an annular geometry.) The Solution (6.19) reduces the PDE problem to an eigenvalue problem in ODE for $\boldsymbol{\Phi}(y)$ with $\lambda$ being the eigenvalue parameter:

$$
\begin{array}{r}
\left(\frac{d^{2}}{d y^{2}}+s_{2}^{2} \lambda^{2}\right)\left(\frac{d^{2}}{d y^{2}}+s_{1}^{2} \lambda^{2}\right) \hat{\boldsymbol{\Phi}}=0 \\
\mathbf{Y}= \pm 1: \quad\left(\alpha_{1} \frac{d^{2}}{d y^{2}}+\lambda^{2}\right) \hat{\Phi}=\left(\alpha_{4} \frac{d^{2}}{d y^{2}}+\alpha_{3} \lambda^{2}\right) \frac{d \hat{\Phi}}{d y}=0 . \quad(6.20 \mathrm{~b}, \mathrm{c})
\end{array}
$$

This problem has two distinct families of solutions. One is even in $z=h y$ and is associated with the bending action of the plate. The eigenfunctions for this family are

$$
\begin{aligned}
\Phi_{B k}(z) \equiv \hat{\Phi}_{B k}(y)= & \left(1-\alpha_{1} s_{1}^{2}\right) \cos \left(s_{1} \bar{\lambda}_{k}^{B} h\right) \cos \left(s_{2} \bar{\lambda}_{k}^{B} z\right) \\
& -\left(1-\alpha_{1} s_{2}^{2}\right) \cos \left(s_{2} \bar{\lambda}_{k}^{B} h\right) \cos \left(s_{1} \bar{\lambda}_{k}^{B} z\right)
\end{aligned}
$$

for $k=1,2, \ldots$, with the corresponding eigenvalues $\left\{\bar{\lambda}_{k}^{B}\right\}$ being the (nonzero) roots of

$$
\frac{\tan \left(s_{1} \bar{\lambda} h\right)}{\tan \left(s_{2} \bar{\lambda} h\right)}=\frac{s_{1}}{s_{2}} .
$$

The other family of solutions is odd in $z$ and is associated with the stretching action of the plate. The eigenfunctions for this family are

$$
\begin{aligned}
\Phi_{S k}(z) \equiv \hat{\Phi}_{S k}(y)= & \left(1-\alpha_{1} s_{1}^{2}\right) \sin \left(s_{1} \bar{\lambda}_{k}^{s} h\right) \sin \left(s_{2} \bar{\lambda}_{k}^{s} z\right) \\
& -\left(1-\alpha_{1} s_{2}^{2}\right) \sin \left(s_{2} \bar{\lambda}_{k}^{s} h\right) \sin \left(s_{1} \bar{\lambda}_{k}^{S} z\right)
\end{aligned}
$$

for $k=1,2,3, \ldots$ with the corresponding eigenvalues $\left\{\bar{\lambda}_{k}^{s}\right\}$ being the (nonzero) roots of

$$
\frac{\tan \left(s_{1} \bar{\lambda} h\right)}{\tan \left(s_{2} \bar{\lambda} h\right)}=\frac{s_{2}}{s_{1}}
$$


As in the plane strain case, we expect

$$
\phi=\phi_{I}+\phi_{R} \equiv \phi_{I}+\sum_{k}\left[a_{k}^{B} \Phi_{B k} I_{0}\left(\bar{\lambda}_{k}^{B} r\right)+a_{k}^{S} \Phi_{S k} I_{0}\left(\bar{\lambda}_{k}^{S} r\right)\right]
$$

The coefficients $\{a, b, \ldots, e\}$ associated with $\boldsymbol{\phi}_{I}$ and $\left\{\boldsymbol{a}_{\boldsymbol{k}}^{\boldsymbol{B}}\right\}$ and $\left\{\boldsymbol{a}_{\boldsymbol{k}}^{\boldsymbol{S}}\right\}$ associated with the residual solution state $\phi_{R}$ are to be determined by the relevant edge data, say at the circular edge $r=r_{0}$.

\section{Five first integrals for axisymmetric deformations}

Integrate Equation (6.lb) across the plate thickness to get

$$
\begin{aligned}
0 & =\int_{-h}^{h}\left[\left(r \sigma_{r z}\right)_{, r}+\left(r \sigma_{z z}\right)_{, z}\right] d z=\int_{-h}^{h}\left(r \sigma_{r z}\right)_{, r} d z+\left[r \sigma_{z z}\right]_{-h}^{h} \\
& =\left[r \int_{-h}^{h} \sigma_{r z} d z\right]_{, r}+\left[r \sigma_{z z}\right]_{-h}^{h} .
\end{aligned}
$$

The term involving $\boldsymbol{\sigma}_{z z}$ vanishes because of the traction-free conditions on the upper and lower face of the plate. Upon integrating Equation (7.1) with respect to $r$, we get a first integral

$$
\int_{-h}^{h} \sigma_{r z} d z=\frac{F_{0}}{r}
$$

where $F_{0}$ is a constant of integration. The overall equilibrium of a circular portion of radius $\mathrm{r}$ of the plate shows that $2 \pi F_{0}$ is the resultant force of the distributed loading in the interior of that plate portion.

Next, we integrate the equilibrium equation (6.la) and the compatibility equation (6.5a) across the thickness to get

$$
\left(r N_{r r}\right)_{, r}=N_{\theta \theta}
$$

and

$$
a_{11} N_{r r}+a_{12} N_{\theta \theta}+a_{13} N_{z z}=\left[r\left(a_{12} N_{r r}+a_{11} N_{\theta \theta}+a_{13} N_{z z}\right)\right]_{, r}
$$

with

$$
\left\{N_{r r}, N_{\theta \theta}, N_{z z}\right\}=\int_{-h}^{h}\left\{\sigma_{r r}, \sigma_{\theta \theta}, \sigma_{z z}\right\} d z
$$

We now use Equation (7.3a) to eliminate $N_{\theta \theta}$ from (7.3b) to get

$$
a_{11}\left\{N_{r r}-\left[r\left(r N_{r r}\right)_{, r}\right]_{, r}\right\}+a_{13}\left\{N_{z z}-\left[r N_{z z}\right]_{, r}\right\}=0 .
$$


With

$$
\begin{aligned}
N_{z z} & =\int_{-h}^{h} \sigma_{z z} d z=\left[z \sigma_{z z}\right]_{-h}^{h}-\int_{-h}^{h} z \sigma_{z z, z} d z \\
& =\int_{-h}^{h} \frac{z}{r}\left(r \sigma_{r z}\right)_{, r} d z \equiv \frac{1}{r}\left(r M_{r z}\right)_{, r}
\end{aligned}
$$

it is possible to write Equation (7.4) as

$$
\left[\frac{d^{2}}{d r^{2}}-\frac{1}{r} \frac{d}{d r}\right]\left[a_{13}\left(r M_{r z}\right)+a_{11}\left(r^{2} N_{r r}\right)\right]=0 .
$$

The solution of this first order linear ordinary differential equation is

$$
N_{r r}+\frac{a_{13}}{a_{11}} \frac{M_{r z}}{r}=\frac{C_{1}}{r^{2}}+C_{2}
$$

it may be written as another first integral

$$
\int_{-h}^{h}\left[\sigma_{r r}+\frac{a_{13}}{a_{11}} \frac{z}{r} \sigma_{r z}\right] d z=\frac{C_{1}}{r^{2}}+C_{2} .
$$

For the third integral, we multiply Equations (6.la) and (6.5a) by $z$ and integrate across the thickness to get

$$
\begin{aligned}
0 & =\int_{-h}^{h}\left[\left(r \sigma_{r r}\right)_{, r}-\sigma_{\theta \theta}+\left(r \sigma_{r z}\right)_{, z}\right] z d z \\
& =\left(r M_{r r}\right)_{, r}-M_{\theta \theta}+\left[\left.r z \sigma_{r z}\right|_{-h} ^{h}-\int_{-h}^{h} r \sigma_{r z} d z\right] \\
& =\left(r M_{r r}\right)_{, r}-M_{\theta \theta}-F_{0}
\end{aligned}
$$

and

$$
\begin{aligned}
0 & =-\int_{-h}^{h}\left[\left(r \epsilon_{\theta \theta}\right)_{, r}-\epsilon_{r r}\right] z d z \\
& =a_{11} M_{r r}+a_{12} M_{\theta \theta}+a_{13} M_{z z}-\left[r\left(a_{12} M_{r r}+a_{11} M_{\theta \theta}+a_{13} M_{z z}\right)\right]_{. r}
\end{aligned}
$$

where

$$
\left\{M_{r r}, M_{\theta \theta}, M_{z z}\right\}=\int_{-h}^{h}\left\{\sigma_{r r}, \sigma_{\theta \theta}, \sigma_{z z}\right\} z d z
$$


Observe that

$$
\begin{aligned}
M_{z z} & \equiv \int_{-h}^{h} \sigma_{z z} z d z=1 /\left.2 z^{2} \sigma_{z z}\right|_{-h} ^{h}-\int_{-h}^{h} 1 / 2 z^{2} \sigma_{z z, z} d z \\
& =\frac{1}{r} \int_{-h}^{h} 1 / 2 z^{2}\left(r \sigma_{r z}\right)_{, r} d z \equiv \frac{1}{r}\left(r S_{r z}\right)_{, r}
\end{aligned}
$$

We now use Equations (7.9) and (7.12) to eliminate $\boldsymbol{M}_{\boldsymbol{\theta} \theta}$ and $M_{z z}$ from (7.10) to get

$$
\frac{d}{d r}\left\{a_{11} r^{3} \frac{d M_{r r}}{d r}+a_{13}\left[r^{2} \frac{d S_{r z}}{d r}-r S_{r 2}\right]\right\}=\left(a_{11}-a_{12}\right) F_{0} r
$$

Upon integrating this relation twice with respect to $r$, we get

$$
a_{11} M_{r r}+a_{13} \frac{S_{r z}}{r}=1 / 2\left(a_{11}-a_{12}\right) F_{0} \ln (r)+\frac{C_{3}}{r^{2}}+C_{4}
$$

which may be written in the form of a first integral:

$$
\text { (iii) } \int_{-\mathrm{h}}^{\mathrm{h}}\left[a_{11} z \sigma_{r r}+1 / 2 r a_{13} z^{2} \sigma_{r z}\right] d z=1 / 2\left(a_{11}-a_{12}\right) F_{0} \ln (r)+C_{4}+\frac{C_{3}}{r^{2}}
$$

For another first integral, we integrate the strain displacement relation $r \boldsymbol{\epsilon}_{\boldsymbol{\theta}}=\boldsymbol{u}_{\boldsymbol{r}}$ to get,

$$
\begin{aligned}
\int_{-h}^{h} u_{r} d z & =r\left\{a_{12} N_{r r}+a_{11} N_{\theta \theta}+a_{13} N_{z z}\right\} \\
& =r\left\{a_{12} N_{r r}+a_{11}\left(r N_{r r}\right)_{, r}+a_{13} r^{-1}\left(r M_{r z}\right)_{, r}\right\} \\
& =\left(a_{12}-a_{11}\right) r N_{r r}+2 a_{11} r C_{2}
\end{aligned}
$$

where we have used Equations (7.3a), (7.5) and (7.7) to express the right-hand side of (7.16) in terms of $N_{r r}$ alone. We may now use Equation (7.16) to eliminate $N_{r r}$ from (7.8) to get the following first integral:

(iv) $\int_{-h}^{h}\left[a_{11} u_{r}+a_{13}\left(a_{12}-a_{11}\right) z \sigma_{r z}\right] d z=a_{11}\left[\frac{C_{1}}{r}\left(a_{12}-a_{11}\right)+C_{2} r\left(a_{12}+a_{11}\right)\right.$ 
For the last integral, we integrate the identity $z \boldsymbol{r} \boldsymbol{\epsilon}_{\boldsymbol{\theta}}=\boldsymbol{z} \boldsymbol{u}_{\boldsymbol{r}}$ across the thickness to get

$$
\begin{aligned}
\int_{-h}^{h} u_{r} z d z= & r\left\{a_{12} M_{r r}+a_{11} M_{\theta \theta}+a_{13} M_{z z}\right\} \\
= & r\left\{a_{12} M_{r r}+a_{11}\left(r M_{r r}\right)_{, r}+a_{13} r^{-1}\left(r S_{r z}\right)_{, r}-a_{11} F_{0}\right\} \\
= & \left(a_{12}-a_{11}\right) r M_{r r}+\left[2 C_{4}-1 / 2\left(a_{11}+a_{12}\right) F_{0}\right] r \\
& +\left(a_{11}-a_{12}\right) F_{0} r \ln (r)
\end{aligned}
$$

where we have used Equations (7.9), (7.12) and (7.14) to eliminate $M_{\theta \theta}, M_{z z}$ and $S_{r z}$. We now use Equation (7.18) to eliminate $M_{r r}$ from (7.15) to get the following first integral:

$$
\begin{aligned}
\int_{-h}^{h}\left[2 a_{11} z u_{r}+a_{13}\left(a_{12}-a_{11}\right) z^{2} \sigma_{r z}\right] d z \\
=\left(a_{11}^{2}-a_{12}^{2}\right) F_{0} r \ln (r)+2\left(a_{12}-a_{11}\right) \frac{c_{3}}{r} \\
\quad+r\left(a_{11}+a_{12}\right)\left(2 C_{4}-a_{11} F_{0}\right) .
\end{aligned}
$$

The integral relations (i)-(v) will be used in the next section to establish the necessary conditions for edge data to induce only a decaying elastostatic state.

\section{N ecessary conditions for an axisymmetric residual state}

For the case where stress data are prescribed along the cylindrical edge $\{\boldsymbol{r}=$ $\left.r_{0},|z| \leq h\right\}$ of a circular plate

$$
\sigma_{r r}\left(r_{0}, z\right)=\bar{\sigma}_{r r}(z), \quad \sigma_{r z}\left(r_{0}, z\right)=\bar{\sigma}_{r z}(z)
$$

we derive below three relevant necessary conditions for the edge data to induce only a residual state.

The constant $\boldsymbol{F}_{0}$ in Equation (7.2) will be shown to vanish for a residual state. With the expression for $\boldsymbol{\sigma}_{\boldsymbol{r} z}$ in (6.7), it suffices to show

$$
\int_{-h}^{h}\left[\alpha_{1} \Phi_{k}^{\prime \prime}+\bar{\lambda}_{k}^{2} \Phi_{k}\right] d z=0
$$

where ()$^{\prime} \equiv d() / d z$ throughout this section. For the bending portion of the 
residual solution, we have from Equation (6.2la)

$$
\begin{aligned}
\int_{-h}^{h}\left[\alpha_{1} \Phi_{B k}^{\prime \prime}+\left(\bar{\lambda}_{k}^{B}\right)^{2} \Phi_{B k}\right] d z & \left(\bar{\lambda}_{k}^{B}\right)^{2}\left(1-\alpha_{1} s_{1}^{2}\right)\left(1-\alpha_{1} s_{2}^{2}\right) \int_{-h}^{h}\left[\cos \left(\bar{\lambda}_{k}^{B} s_{1} h\right) \cos \left(\bar{\lambda}_{k}^{B} s_{2} z\right)\right. \\
& \left.\quad-\cos \left(\bar{\lambda}_{k}^{B} s_{2} h\right) \cos \left(\bar{\lambda}_{k}^{B} s_{1} z\right)\right] d z \\
= & 2 \bar{\lambda}_{k}^{B}\left(1-\alpha_{1} s_{1}^{2}\right)\left(1-\alpha_{1} s_{2}^{2}\right) \cos \left(\bar{\lambda}_{k}^{B} s_{1} h\right) \cos \left(\bar{\lambda}_{k}^{B} s_{2} h\right) \\
& \times\left\{\frac{1}{s_{2}} \tan \left(\bar{\lambda}_{k}^{B} s_{2} h\right)-\frac{1}{s_{1}} \tan \left(\bar{\lambda}_{k}^{B} s_{1} h\right)\right\} .
\end{aligned}
$$

The right-hand side of the above relation vanishes because of (6.2lb). The proof of Equation (8.2) for the stretching portion of the residual solution is trivial as the integrand is an odd function of $\mathrm{z}$ for this case. It follows that the integrated equilibrium condition (7.2) becomes

$$
\int_{-h}^{h} \sigma_{r z}^{I} d z=\frac{F_{0}}{r}
$$

as the integral of $\boldsymbol{\sigma}_{r z}^{\boldsymbol{R}}$ across the thickness vanishes by (8.2). Furthermore, a straightforward calculation using $(6.18 \mathrm{~g})$ gives

$$
F_{0}=4 / 3 h^{3} c\left(\alpha_{4}-\alpha_{1} \alpha_{3}\right),
$$

where $\mathrm{c}$ is a constant associated with $\boldsymbol{\phi}_{c}$ in the interior solution (6.18) for $\boldsymbol{\phi}$.

Next, we will show that the constants $C_{1}$ and $C_{2}$ in Equation (7.8) must also vanish for a residual state. Evidently, we need only to do this for a stretching portion of the solution as the integrand is an odd function of $z$ for the bending problem. Now, for a residual stress state, we have from Equations (6.7) and (6.22a)

$$
\begin{aligned}
& \int_{-h}^{h}\left[\sigma_{r r}^{R}+\frac{a_{13}}{a_{11}} \frac{z}{r} \sigma_{r z}^{R}\right] d z \\
& =-\int_{-h k}^{h} \sum_{k} a_{k}^{s}\left\{\left[\Delta_{r}+\alpha_{1} \frac{\partial^{2}}{\partial z^{2}}\right] \Phi_{S k}^{\prime} I_{0}\left(\bar{\lambda}_{k}^{s} r\right)-\frac{1-\alpha_{2}}{r} \Phi_{S k}^{\prime} I_{0, r}\left(\bar{\lambda}_{k}^{S} r\right)\right. \\
& \left.\quad+\frac{a_{13} z}{a_{11} r}\left[\left(\bar{\lambda}_{k}^{S}\right)_{r}^{2}+\alpha_{1} \frac{\partial^{2}}{\partial z^{2}}\right] \Phi_{S k} I_{0, r}\left(\bar{\lambda}_{k}^{S} r\right)\right\} d z \\
& =-\sum_{k} a_{k}^{s}\left\{\left.\left[\left(\bar{\lambda}_{k}^{S}\right)^{2}+\alpha_{1} \frac{\partial^{2}}{\partial z^{2}}\right] \Phi_{S k}\right|_{-h} ^{h} I_{0}\left(\bar{\lambda}_{k}^{S} r\right)\right. \\
& \left.\quad+\frac{1}{r} I_{0, r}\left(\bar{\lambda}_{k}^{s} r\right) \int_{-h}^{h}\left[\frac{a_{13}}{a_{11}} z\left(\left(\bar{\lambda}_{k}^{s}\right)^{2}+\alpha_{1} \frac{\partial^{2}}{\partial z^{2}}\right)-\left(1-\alpha_{2}\right) \frac{\partial}{\partial z}\right] \Phi_{S k} d z\right\}
\end{aligned}
$$


The term evaluated at the faces vanishes by the boundary condition (6.20b). It may be verified by the direct substitution of the solution (6.22) and (6.8) that the integral on the right side of Equation (8.6) is also zero.

A similar argument shows that the constants $C_{3}$ and $C_{4}$ in (7.14) must also vanish for a residual state.

The first integrals (7.2), (7.8) and (7.15) hold for any circular ring of the plate. In particular, they hold at the edge $r=r_{0}$. Therefore, we have the following result for the stress data case:

\section{Stress Data:}

For the stress edge data to induce only a residual elastostatic state, the axisymmetric edge tractions $\overline{\boldsymbol{\sigma}}_{r r}(z)$ and $\overline{\boldsymbol{\sigma}}_{r z}(z)$ must satisfy the following three (necessary) conditions:

$$
\begin{array}{r}
\int_{-h}^{h} \bar{\sigma}_{r z} d z=0, \quad \int_{-h}^{h}\left[a_{11} \bar{\sigma}_{r r}+\frac{1}{r} a_{13} z \bar{\sigma}_{r z}\right] d z=0 \\
\int_{-h}^{h}\left[a_{11} z \bar{\sigma}_{r r}+1 / 2 r a_{13} z^{2} \bar{\sigma}_{r z}\right] d z=0 .
\end{array}
$$

For isotropic materials, the above result is identical to the corresponding result obtained in references 8 and 9 , but now the theorem holds without the asymptoticity (and isotropy) restriction. The last two conditions in Equations (8.7) emphasize that Saint Venant's principle is generally not applicable to axisymmetric deformation of circular plates. The conventional stress boundary conditions for plate theories with $N_{r r}$ and $M_{r r}$ (as well as transverse shear resultant $Q_{r}$ ) prescribed at an edge (see $[17,21]$ for example) should only be used for very thin plates and $\overline{\boldsymbol{\sigma}}_{r r}$ not small compared to $\overline{\boldsymbol{\sigma}}_{r z}$.

Three relevant necessary conditions for a residual state can also be established for the mixed data set:

$$
u_{r}\left(r_{0}, z\right)=\bar{u}_{r}(z), \quad \sigma_{r z}\left(r_{0}, z\right)=\bar{\sigma}_{r z}(z)
$$

We have already shown that the five constants $F_{0}, C_{1}, C_{2}, C_{3}$ and $C_{4}$ must vanish for a residual state. Upon setting these constants equal to zero and $r=r_{0}$ in Equations (7.2), (7.17) and (7.19), we get the following result:

\section{Mixed Data}

If the mixed edge data (8.8) induces only a residual elastostatic state, $\overline{\boldsymbol{\sigma}}_{z}(z)$ and $\bar{u}_{r}(z)$ must satisfy the following three (necessary) conditions:

$$
\begin{gathered}
\int_{-h}^{h} \bar{\sigma}_{r z} d z=0, \quad \int_{-h}^{h}\left[a_{11} \bar{u}_{r}+a_{13}\left(a_{12}-a_{11}\right) z \bar{\sigma}_{r z}\right] d z=0 \\
\int_{-h}^{h}\left[2 a_{11} z \bar{u}_{r}+a_{13}\left(a_{12}-a_{11}\right) z^{2} \bar{\sigma}_{r z}\right] d z=0 .
\end{gathered}
$$


corresponding conditions obtained in [8] for isotropic materials. The second is for plate extension and reduces to the corresponding condition in [9] for isotropic materials. The more general results of Equation (8.9) are for transversely isotropic materials and without the asymptoticity restriction.

\section{Boundary conditions for the interior solution}

The necessary conditions for a residual state developed in Sections 5 and 8 can be converted into a set of boundary conditions appropriate for the interior solution or its various thin and thick plate theory approximations. Unlike previous attempts $[1,3,4,18,19]$, these boundary conditions do not involve the boundary layer solution components. As the difference between the exact solution and the interior solution is a residual solution state, our necessary conditions apply to this difference evaluated at an edge. Cases for which exact necessary conditions are not obtained in this paper will be discussed elsewhere $[15,16]$.

\section{A. Plane strain deformations}

(a) Case (1) (stress data). Consider the residual solution

$$
\begin{aligned}
& \tilde{\sigma}_{x x} \equiv \sigma_{x x}-\sigma_{x x}^{I}=\sigma_{x x}-\left[\frac{N_{0}}{2 h}+\frac{3 M_{0}}{2 h^{3}} z+\frac{3 Q_{0}}{2 h^{3}} x z\right] \\
& \tilde{\sigma}_{x z} \equiv \sigma_{x z}-\sigma_{x z}^{I}=\sigma_{x z}-\frac{3 Q_{0}}{4 h^{3}}\left(h^{2}-z^{2}\right)
\end{aligned}
$$

where $\boldsymbol{\sigma}_{x x}^{I}$ and $\boldsymbol{\sigma}_{x z}^{I}$ have been taken from Equation (3.7). For an edge $x=l, \tilde{\boldsymbol{\sigma}}_{x \boldsymbol{x}}(\boldsymbol{l}, \boldsymbol{z})$ and $\tilde{\sigma}_{x z}(l, z)$ may be treated as the edge data which induce the residual state $\tilde{\boldsymbol{\sigma}}_{x x}(x, z)$ and $\tilde{\boldsymbol{\sigma}}_{x z}(x, z)$. Hence, the three conditions (5.1) apply to $\tilde{\boldsymbol{\sigma}}_{x x}(l, z)$ and $\tilde{\boldsymbol{\sigma}}_{x z}(l, z)$; they give

$$
\begin{aligned}
& \int_{-h}^{h}\left[\boldsymbol{\sigma}_{x x}^{I}\right]_{x=l} d z=N_{0}=\int_{-h}^{h} \overline{\boldsymbol{\sigma}}_{x x} d z \\
& \int_{-h}^{h}\left[\boldsymbol{\sigma}_{x z}^{I}\right]_{x=l} d z=Q_{0}=L_{-\mathrm{h}}^{h} \overline{\boldsymbol{\sigma}}_{x z} d z \\
& \int_{-h}^{h}\left[z \boldsymbol{\sigma}_{x x}^{I}\right]_{x=l} d z=M_{0}+Q_{0} l=\int_{-h}^{h} \overline{\boldsymbol{\sigma}}_{x x} z d z .
\end{aligned}
$$

The three relations (9.2) determine the interior solution up to a rigid motion characterized by the remaining three constants c, $d$ and $\boldsymbol{\omega}$ which appear only in the displacement components in (3.9). Three (integrated) displacement conditions are needed at a second edge (or any station along the length of the strip) to completely determine these constants. If stress data are prescribed at the second edge, then they must give rise to the same stress resultants $N_{0}$ and $Q_{0}$ and the stress couple $\boldsymbol{M}_{0}$. As expected, the constants c, $d$ and $\boldsymbol{\omega}$ will remain unspecified 
(b) Case (2) (mixed data). The three conditions in (5.8), applied to the edge data at $x=l$ :

$$
\tilde{\sigma}_{x z}(l, z)=\left[\sigma_{x z}-\sigma_{x z}^{I}\right]_{x=l}, \quad \tilde{u}_{x}(l, z)=\left[u_{x}-u_{x}^{I}\right]_{x=l}
$$

where $\boldsymbol{u}_{\boldsymbol{x}}^{\boldsymbol{I}}$ is defined by (3.9a), give

$$
\begin{aligned}
\int_{-h}^{h}\left[\sigma_{x z}^{I}\right]_{x=l} d z & =Q_{0}=\int_{-h}^{h} \bar{\sigma}_{x z} d z \\
\int_{-h}^{h}\left[u_{x}^{I}-a_{12} z \sigma_{x z}^{I}\right]_{x=l} d z & =\frac{N_{o}}{E_{x_{1}}}+2 h d=\int_{-h}^{h}\left(\bar{u}_{x}+\frac{\nu}{E} z \bar{\sigma}_{x z}\right) d z \\
\int_{-h}^{h}\left[z u_{x}^{I}-\frac{1}{2} a_{12} z^{2} \sigma_{x z}^{I}\right]_{x=l} d z & =\frac{M_{o^{\prime}}}{E_{1}}-\frac{2 h^{3} \omega}{3}+Q_{0}\left[\frac{o^{2}}{2 E_{1}}+\frac{h^{2}}{10}\left(\frac{2 \nu}{E}-\frac{1}{G}\right)\right] \\
& =\int_{-h}^{h}\left[z \bar{u}_{x}+\frac{\nu}{2 \mathrm{E}} z^{2} \bar{\sigma}_{x z} \mid d z\right.
\end{aligned}
$$

Three other conditions are needed at a second edge to completely determine the six constants $N_{0}, Q_{0}, M_{0}, c, d$ and $\boldsymbol{\omega}$. To obtain the appropriate edge conditions for a particular (thin or thick) plate theory, we should expand both sides of the relations in (9.4) in powers of $h / l$ and retain a suitable number of terms in these expansions. This observation holds for all cases discussed in this section though the parameter $h / l$ does not appear explicitly in case (1).

(c) Case (3) (mixed data). The three conditions in (5.10), applied to the edge data at $\mathrm{x}=\boldsymbol{l}$,

$$
\tilde{\boldsymbol{\sigma}}_{x x}(l, z)=\left[\sigma_{x x}-\sigma_{x x}^{I}\right]_{x=l}, \quad \tilde{u}(l, z)=\left[u_{z}-u_{z}^{I}\right]_{x=l}
$$

where $\boldsymbol{u}_{z}^{I}$ is defined by Equation (3.9b), gives

$$
\begin{aligned}
\int_{-h}^{h}\left[\sigma_{x x}^{I}\right]_{x=l} d z= & N_{0}=\int_{-h}^{h} \bar{\sigma}_{x x} d z \\
\int_{-h}^{h}\left[\sigma_{x x}^{I} z\right]_{x=l} d z= & M_{0}+Q_{0} l \\
= & \int_{-h}^{h} \bar{\sigma}_{x x} z d z \int_{-h}^{h}\left[\left(h^{2}-z^{2}\right) u_{z}^{I}+\frac{1}{3}\left(a_{33}+a_{12}\right) z^{3} \sigma_{x x}^{I}\right]_{x=l} d z \\
= & M_{0}\left[\frac{h^{2}}{5}\left(\frac{1}{G}-\frac{2 \nu}{E}\right)-\frac{l^{2}}{E_{1}}\right]+Q_{0}\left[\frac{h^{2} l}{5}\left(\frac{6}{G}-\frac{2 \nu}{E}\right)-\frac{l^{3}}{3 E_{1}}\right] \\
& +\frac{4 h^{3}}{3}(c+\omega l) \\
= & \int_{-h}^{h}\left[\left(h^{2}-z^{2}\right) \bar{u}_{z}+\frac{1}{3}\left(\frac{1}{G}-\frac{\nu}{E}\right) \bar{\sigma}_{x x} z^{3}\right] d z
\end{aligned}
$$


Three other conditions at a second edge are needed to completely determine the six unknown constants in the interior solution.

\section{B. Axisymmetric deformations}

(a) Stress data. The three conditions in (8.7), applied to the edge data at $r=r_{0}$ of a circular plate:

$$
\tilde{\sigma}_{r r}\left(r_{0}, z\right)=\left[\sigma_{r r}-\sigma_{r r}^{I}\right]_{r=r_{0}}, \quad \tilde{\sigma}_{r z}\left(r_{0}, z\right)=\left[\sigma_{r z}-\sigma_{r z}^{I}\right]_{r=r_{0}}
$$

where $\boldsymbol{\sigma}_{r r}^{I}$ and $\boldsymbol{\sigma}_{r z}^{I}$ are defined in (6.18), give

$$
\begin{gathered}
\int_{-h}^{h}\left[\sigma_{r z}^{I}\right]_{r=r_{0}} d z=\int_{-{ }_{h}^{h}}^{h} \bar{\sigma}_{r z} d z \\
\int_{-h}^{h}\left[a_{11} \sigma_{r r}^{I}+a_{13} \frac{z}{r} \sigma_{r z}^{I}\right]_{r=r_{0}} d z=\int_{-h}^{h}\left[a_{11} \bar{\sigma}_{r r}+a_{13} \frac{z}{r_{0}} \bar{\sigma}_{r z}\right] d z \\
\int_{-h}^{h}\left[a_{11} z \sigma_{r r}^{I}+a_{13} \frac{z^{2}}{2 r} \sigma_{r z}^{I}\right]_{r=r_{0}} d z=\int_{-h}^{h}\left[a_{11} z \bar{\sigma}_{r r}+a_{13} \frac{z^{2}}{2 r_{0}} \bar{\sigma}_{r z}\right] d z .
\end{gathered}
$$

After we carry out the integration involved, Equation (9.8) becomes three simultaneous linear equations for the constants $\{a, b, c, d, e\}$ in $\boldsymbol{\sigma}_{r r}^{I}$ and $\boldsymbol{\sigma}_{r z}^{I}$. Three other conditions at another edge (or at the center of the plate) are needed to completely determine these five constants and the constant $w_{0}$ associated with a transverse rigid body displacement. If stress data are prescribed at the second edge, the transverse shear stress there must give rise to the same resultants as $\overline{\boldsymbol{\sigma}}_{r z}$ at $\boldsymbol{r}_{\mathbf{0}}$. As expected, $\boldsymbol{w}_{0}$ will be left unspecified in that case.

(b) Mixed data. We apply for this case the three conditions in (8.9) to the edge data

$$
\tilde{\sigma}_{r z}\left(r_{0}, z\right)=\left[\sigma_{r z}-\sigma_{r z}^{I}\right]_{r=r_{0}}, \quad \tilde{u}_{r}\left(r_{0}, z\right)=\left[u_{r}-u_{r}^{I}\right]_{r=r_{0}}
$$

where $\boldsymbol{u}_{\boldsymbol{r}}^{I}$ is calculated from $\boldsymbol{u}_{\boldsymbol{r}}=\boldsymbol{r} \boldsymbol{\epsilon}_{\theta \theta}$, the stress strain relation for $\boldsymbol{\epsilon}_{\theta \boldsymbol{\theta}}$ and the expressions for $\boldsymbol{\sigma}_{r r}^{I}$ and $\boldsymbol{\sigma}_{\theta \theta}^{I}$ in Equation (6.18). We get the following three boundary conditions for the interior solution:

$$
\begin{gathered}
\int_{-h}^{h}\left[\sigma_{r z}^{I}\right]_{r=0} d z=\int_{-h}^{h} \bar{\sigma}_{r z} d z \\
\int_{-h}^{h}\left[a_{11} u_{r}^{I}+a_{13}\left(a_{12}-a_{11}\right) z \sigma_{r z}^{I}\right]_{r=r_{0}} d z=\int_{-h}^{h}\left[a_{11} \bar{u}_{r}+a_{13}\left(a_{12}-a_{11}\right) z \bar{\sigma}_{r z}\right] d z \\
\int_{-h}^{h}\left[2 a_{11} z u_{r}^{I}+a_{13}\left(a_{12}-a_{11}\right) z^{2} \sigma_{r z}^{I}\right]_{r=r_{0}} d z=\int_{-h}^{h}\left[2 a_{11} z \bar{u}_{r}+a_{13}\left(a_{12}-a_{11}\right) z^{2} \bar{\sigma}_{r z}\right] d z .
\end{gathered}
$$


Three more conditions at another edge (or at the center of the plate) are needed to completely determine the six unknown constants in the interior solution.

As mentioned in conjunction with Case (2) of the plane strain problems, the corresponding boundary conditions for a given plate theory are obtained by expanding in powers of $h / l$ all terms in all three necessary conditions deduced in this section for each type of edge data and retaining only a suitable number of terms in each expansion.

\section{Concluding remarks}

We have not obtained similar results on necessary conditions for the pure displacement edge data case in plane strain deformations, or for what were designated as case (B) and case (D) in [8] for axisymmetric deformations of circular plates. Given that no elementary state (2) exists for these cases in the context of the approach used in [7-10], it is not likely that the desired results are forthcoming. In contrast, we do expect that the asymptoticity restriction can be removed from the necessary conditions already obtained for some cases involving edgewise nonuniform data such as cases (B) and (C) for a semi-infinite plate analyzed in [8].

Applications of the results obtained in this paper beyond those given in Section 9, can be found in [15] and [16].

\section{Appendix \\ The interior solution with axisymmetry for transversely isotropic plates}

In this appendix, we sketch the derivation of the interior (Levy-type) solution given by Equation (6.18). Evidently, we need only to consider $\boldsymbol{\phi}_{\boldsymbol{k}}$ for even $\boldsymbol{k}$ in (6.15)-(6.17) as the odd $\boldsymbol{k}$ case is identical. The bending and stretching problems will be discussed separately for clarity.

\section{The plate bending problem}

The general solution of Equation (6.15) for $\boldsymbol{k}=\boldsymbol{0}$ is

$$
\phi_{0}=\frac{1}{6} R_{03}(x) y^{3}+\frac{1}{2} R_{02}(x) y^{2}+R_{01}(x) y+R_{00}(x) \text {. }
$$

The portion corresponding to plate bending is even in the thickness coordinate $y$ (or z)

$$
\phi_{0}=\frac{1}{2} R_{02}(x) y^{2}+R_{00}(x) .
$$

The boundary condition (6.17) for $\boldsymbol{k}=\boldsymbol{0}$ are automatically satisfied and the boundary conditions in (6.16) require $\boldsymbol{R}_{\mathbf{0 2}}(x)$ to be constant. The constant can be set to zero since the function $y^{2}$ gives no contribution to stresses. Thus, we have

$$
\phi_{0}=R_{00}(x)
$$


where the function $\boldsymbol{R}_{\mathbf{0}}(x)$ is to be determined. For $\boldsymbol{k}=2$, the BVP (6.15)-(6.17) has the following solution for the bending problem:

$$
\phi_{2}=\frac{1}{2} R_{22}(x) y^{2}+R_{20}(x)
$$

with

$$
\alpha_{1} R_{22}(x)+\Delta_{x} R_{00}(x)=A_{1}
$$

where $A_{1}$ is a constant and can be set to zero as it does not contribute to the stress components. Thus, we have

$$
\phi_{2}=-\frac{1}{2 \alpha_{1}} \Delta_{x} R_{00}(x) y^{2}+R_{20}(x)
$$

where $R_{20}(x)$ is to be determined. Upon substituting Equations (A.3) and (A.6) into (6.15)-(6.17) for $\boldsymbol{k}=4$, we obtain

$$
\begin{aligned}
\alpha_{4} \phi_{4, y y y y} & =\frac{\alpha_{3}}{\alpha_{1}} \Delta_{x} \Delta_{x} R_{00}(x) \\
y=1: \quad \alpha_{4} \phi_{4, y y y y} & =\frac{\alpha_{3}}{\alpha_{1}} \Delta_{x} \Delta_{x} R_{00}(x), \\
\alpha_{1} \phi_{4, x y y} & =1 / 2 \alpha_{1} \Delta_{x} \Delta_{x} R_{00}^{\prime}(x)-\Delta_{x} R_{20}^{\prime}(x) .
\end{aligned}
$$

The solution of this system is

$$
\phi_{4}=\frac{\alpha_{3}}{24 \alpha_{1} \alpha_{4}} \Delta_{x} \Delta_{x} R_{00}(x) y^{4}+1 / 2 R_{42}(x) y^{2}+R_{40}(x)
$$

with

$$
R_{42}(x)=\frac{\alpha_{4}-\alpha_{3} \alpha_{1}}{2 \alpha_{1}^{2} \alpha_{4}} \Delta_{x} \Delta_{x} R_{00}(x)-\frac{1}{\alpha_{1}} \Delta_{x} R_{20}(x)+A_{2}
$$

where the constant $\boldsymbol{A}_{\mathbf{2}}$ can be omitted. After eliminating $\boldsymbol{R}_{\mathbf{4 2}}$ from Equation (A.9), we get,

$$
\begin{aligned}
\phi_{4}= & \frac{\alpha_{3}}{24 \alpha_{1} \alpha_{4}} \Delta_{x} \Delta_{x} R_{00}(x) y^{4} \\
& +\left\{\frac{\alpha_{4}-\alpha_{1} \alpha_{3}}{4 \alpha_{1}^{2} \alpha_{4}} \Delta_{x} \Delta_{x} R_{00}(x)-1 / 2 \alpha_{1} \Delta_{x} R_{20}(x)\right\} y^{2}+R_{40}(x) .
\end{aligned}
$$

In order to get an equation for $\boldsymbol{R}_{\mathbf{0}}(\boldsymbol{x})$, we solve the Equation (6.15) for $\boldsymbol{k}=6$ to 
get,

$$
\begin{aligned}
\phi_{6}= & \frac{\alpha_{4}-\alpha_{3}\left(\alpha_{1}+\alpha_{3}\right)}{720 \alpha_{1} \alpha_{4}^{2}} \Delta_{x} \Delta_{x} \Delta_{x} R_{00}(x) y^{6} \\
& +\left\{\frac{\left(\alpha_{1}+\alpha_{3}\right)\left(\alpha_{1} \alpha_{3}-\alpha_{4}\right)}{48 \alpha_{1}^{2} \alpha_{4}^{2}} \Delta_{x} \Delta_{x} \Delta_{x} R_{00}(x)+\frac{\alpha_{3}}{24 \alpha_{1} \alpha_{4}} \Delta_{x} \Delta_{x} R_{20}(x) y^{4}\right. \\
& +1 / 2 R_{62}(x) y^{2}+R_{60}(x)
\end{aligned}
$$

The boundary conditions in Equations (6.16) and (6.17) for $k=6$ require

$$
\Delta_{x} \Delta_{x} \Delta_{x} R_{00}(x)=0
$$

and

$$
R_{62}(x)=-\frac{1}{\alpha_{1}} \Delta_{x} R_{40}(x)-\frac{\alpha_{1} \alpha_{3}-\alpha_{4}}{2 \alpha_{1}^{2} \alpha_{4}} \Delta_{x} \Delta_{x} R_{20}(x)
$$

They in turn simplify $\phi_{6}$ to

$$
\begin{aligned}
\phi_{6}= & \frac{\alpha_{3}}{24 \alpha_{1} \alpha_{4}} \Delta_{x} \Delta_{x} R_{20}(x) y^{4} \\
& +\left\{\frac{\alpha_{4}-\alpha_{1} \alpha_{3}}{4 \alpha_{1}^{2} \alpha_{4}} \Delta_{x} \Delta_{x} R_{20}(x)-1 / 2 \alpha_{1} \Delta_{x} R_{40}(x)\right\} y^{2}+R_{60}(x) .
\end{aligned}
$$

Now, the similarity between $\boldsymbol{\phi}_{4}$ and $\boldsymbol{\phi}_{6}$ makes it possible to write the whole solution for $\phi$ as:

$$
\begin{aligned}
\phi & =\left\{\phi_{0}+\epsilon^{2} \phi_{2}+\epsilon^{4} \phi_{4}+\epsilon^{6} \phi_{6}+\cdots\right\}+\epsilon\left\{\phi_{1}+\epsilon^{2} \phi_{3}+\cdots\right\} \\
& \equiv \phi_{\text {even }}+\epsilon \phi_{\text {odd }} .
\end{aligned}
$$

For $\phi_{\text {even }}$, we have

$$
\begin{aligned}
\phi_{\text {even }}= & \left\{R_{00}(x)+\epsilon^{2} R_{20}(x)+\epsilon^{4} R_{40}(x)+\epsilon^{6} R_{60}(x)+\cdots\right\} \\
+ & \frac{\epsilon^{2} z^{2}}{2 h^{2}}\left\{-1 / \alpha_{1}\left[\Delta_{x} R_{00}(x)+\epsilon^{2} \Delta_{x} R_{20}(x)+\epsilon^{4} \Delta_{x} R_{40}(x)+\cdots\right]\right. \\
& \left.\quad+\frac{\alpha_{4}-\alpha_{1} \alpha_{3}}{2 \alpha_{1}^{2} \alpha_{4}} \epsilon^{2}\left[\Delta_{x} \Delta_{x} R_{00}(x)+\epsilon^{2} \Delta_{x} \Delta_{x} R_{20}(x)+\cdots\right]\right\} \\
+ & \frac{\epsilon^{4} z^{4}}{24 h^{4}} \frac{\alpha_{3}}{\alpha_{1} \alpha_{4}}\left\{\Delta_{x} \Delta_{x} R_{00}(x)+\epsilon^{2} \Delta_{x} \Delta_{x} R_{20}(x)+\cdots\right\}
\end{aligned}
$$


where

$$
\Delta_{x} \Delta_{x} \Delta_{x} R_{j_{0}}(x)=0
$$

It is not difficult to see that $\phi_{\text {odd }}$ has the same structure as $\phi_{\text {even }}$ with $\boldsymbol{R}_{\mathbf{0 0}}, \boldsymbol{R}_{\mathbf{2 0}}, \boldsymbol{R}_{\mathbf{4 0}}$; etc., replaced by $R_{10}, R_{30}, R_{50}$, etc.

Let

$$
R_{0}(x)=\sum_{k=o}^{\infty} R_{k 0}(x) \epsilon^{k}
$$

Evidently, we have $\Delta_{x} \Delta_{x} \Delta_{x} R_{0}(x)=0$. The stress function $\phi$ can then be written as

$$
\begin{aligned}
\phi= & R_{0}(x)+\frac{\epsilon^{2} z^{2}}{2 h^{2}}\left\{-1 / \alpha_{1} \Delta_{x} R_{0}(x)+\frac{\alpha_{4}-\alpha_{1} \alpha_{3}}{2 \alpha_{1}^{2} \alpha_{4}} \epsilon^{2} \Delta_{x} \Delta_{x} R_{0}(x)\right\} \\
& +\frac{\epsilon^{4} z^{4}}{24 h^{4}} \frac{\alpha_{3}}{\alpha_{1} \alpha_{4}} \Delta_{x} \Delta_{x} R_{0}(x)
\end{aligned}
$$

or

$$
\phi=R_{0}(r)-\frac{z^{2}}{2 \alpha_{1}} \Delta_{r} R_{0}(r)+\frac{\alpha_{4}-\alpha_{1} \alpha_{3}}{4 \alpha_{1}^{2} \alpha_{4}} z^{2} h^{2} \Delta_{r} \Delta_{r} R_{0}(r)+\frac{\alpha_{3}}{\alpha_{1} \alpha_{4}} \frac{z^{4}}{24} \Delta_{r} \Delta_{r} R_{0}(r)
$$

where $\Delta_{r} \Delta_{r} \Delta_{r} R_{0}(r)=0$ with $\Delta_{r}=()_{, r r}+r^{-1}()_{, r}$.

The general solution for $R_{0}(r)$ is

$$
R_{0}(r)=C_{1}+C_{2} \ln r+C_{3} r^{2}+a r^{2} \ln r+b r^{4}+c r^{4} \ln r
$$

where $C_{1}, C_{2}$ and $C_{3}$ may be set to zero since they give no contributions to stresses. It follows that the interior solution for the bending problem is as given by the first three terms of Equation (6.18). This solution reduces to the known result for the isotropic case upon specialization.

\section{The plate stretching problem}

The interior solution for the stretching problem is derived similarly. The boundary conditions (6.16) and (6.17) for $k=O$ reduce the portion of Equation (A.1) odd in $\mathrm{Y}$ to

$$
\phi_{0}=y R_{01}(x)
$$


where $R_{01}(x)$ is to be determined. For $\boldsymbol{k}=2$, we have from Equations (6.15)-(6.17)

$$
\phi_{2}=\frac{y^{5}}{6} R_{23}(x)+y R_{21}(x)
$$

with

$$
\alpha_{4} R_{23}(x)+\alpha_{3} \Delta_{x} R_{01}(x)=0, \quad \alpha_{1} R_{23}^{\prime}(x)+\Delta_{x} R_{01}^{\prime}(x)=0 .
$$

It follows from Equation (A.24b) that $R_{23}(x)=-\alpha_{3} \Delta_{x} R_{01}(x) / \alpha_{4}=-\alpha_{3} \gamma_{0} / \alpha_{4}$ where $\gamma_{0}$ is a constant of integration and therewith

$$
+2=-\frac{y_{-}^{3} a_{3}}{6} \frac{\alpha_{4}}{\alpha_{4}} \gamma_{0}+y R_{21}(x)
$$

For $\boldsymbol{k}=4$, we have from Equations (6.15)-(6.17)

$$
\begin{gathered}
\phi_{4}=\frac{y^{3}}{6} R_{43}(x)+y R_{41}(x) \\
\alpha_{4} R_{43}(x)+\alpha_{3} \Delta_{x} R_{21}(x)=0, \quad \alpha_{1} R_{43}^{\prime}(x)+\Delta_{x} R_{21}^{\prime}(x)=0 .
\end{gathered}
$$

Similar to the $k=2$ case, we get

$$
\phi_{4}=-\frac{y^{3}}{6} \frac{\alpha_{3}}{\alpha_{4}} \gamma_{2}+y R_{41}(x)
$$

with $\Delta_{x} R_{21}(x)=\gamma_{2}$ where $\gamma_{2}$ is a constant.

Now, the solution for $\phi$ can be written as

$$
\begin{aligned}
\phi & =\left\{\phi_{0}+\epsilon^{2} \phi_{1}+\epsilon^{4} \phi_{2}+\cdots\right\}+\epsilon\left\{\phi_{1}+\epsilon^{2} \phi_{3}+\cdots\right\} \\
& \equiv \phi_{\text {even }}+\epsilon \phi_{\text {odd }}
\end{aligned}
$$

$\phi_{\text {even }}=-\frac{y^{3}}{6} \frac{\alpha_{3}}{\alpha_{4}} \epsilon^{2}\left\{\gamma_{0}+\epsilon^{2} \gamma_{2}+. . \mid\right\}+y\left\{R_{01}(x)+\epsilon^{2} R_{21}(x)+\epsilon^{4} R_{41}(x)+\mid.\right\}$

where $R_{01}(x), R_{21}(x)$, etc., can be easily determined from $\Delta_{x} R_{j 1}=\gamma_{j}$ to be

$$
R_{j 1}(x)=\frac{\gamma_{j}}{4} x^{2}+\delta_{j} \ln x+C_{j}
$$

The constants $\left\{C_{i}\right\}$ will be set to zero as they do not contribute to the stresses. 
The structure of $\phi_{\text {odd }}$ is evidently the same as $\phi_{\text {even }}$. The series for the plate stretching portion of the interior solution may be summed to give the last two terms of Equation (6.18). This solution reduces to the known result for the isotropic case upon specialization.

\section{Acknowledgments}

Research supported by the University of British Columbia, an I. W. Killam fellowship, and by NSF grant number DMS-8606198.

\section{R eferences}

1. K. 0. FRIEDRICHS and R. F. DRESSLER, A boundary layer theory of elastic bending of plates, Comm. Pure Appl. Math. 14:1-33 (1961).

2. S. GERMAIN, Recherches sur la theorie de surfaces elastiques, Mme. V. Courcier Paris, 1821.

3. A. L. Gol'DenVEIZER, Derivation of an approximate theory of bending of a plate by the method of asymptotic integration of the equations of theory of elasticity, P.M.M.26:668-686 (1962).

4. A. L. GOL'DENVEIZER and A. V. KOLOS, On the derivation of two-dimensional equations in the theory of thin elastic plates, P.M. M. 29:141-155 (1965).

5. R. D. GREGORY Green's functions, bi-linear forms and completeness of the Papkovich-Fadle eigenfunctions, J. Elasticity 10:295-327 (1980).

6. R. D. GREGORY The traction boundary value problem for the elastostatic semi-infinite strip, existence of solution and completeness of the Papkovich-Fadle Eigenfunctions,. Elasticity 10:295-327 (1980).

7. R. D. GREGORY and F. Y. M. WAN, Decaying states of plane strain in a semi-infinite strip and boundary conditions for plate theory, J. Elasticity 14:27-64 (1984).

8. R. D. Gregory and F. Y. M. WAN, On plate theories and Saint-Venant's principle,Int. J. Solids Structures 21:1005-1024 (1985).

9. R. D. GReGORY and F. Y. M. WAN, Edge effect in the stretching of plates, Local Effects in the Analysis of Structures, ed. P. Ladevèze, Elsevier Science Publishers B.V., Amsterdam, 1985, 35-54.

10. R. D. GREGORY and F. Y. M. WAN, On the interior solution for linearly elastic plate, J. Appl. Mech. 55, to appear in 1988.

11. G. KiRchHOFF, Über das gleichewicht und die bewegung einer elastischen scheibe, J. Reine Ange. Math. (Crelle) 40:51 (1850).

12. M. LEvy, Memoire sur la theorie des plaques elastique planes, J. Math. Pure Appl. 30:219-306 (1877).

13. S. G. LEKHNITSKII, Theory of Elasticity of an Anisotropic Body, Holden-Day Inc., San Francisco, 1963.

14. Y. H. Lin, A mathematical theory of elastic orthotropic plates in plane strain and axisymmetric deformations, Ph.D. dissertation, Institute of Appl. Math., University of British Columbia, Vancouver, 1987.

15. Y. H. LiN and F. Y. M. WAN, Extension, bending and flexure of an orthotropic semi-infinite cantilever strip and the foundations of plate theories, Tech. Report 87-6, Dept. of Appl. Math., University of Washington, Seattle, July, 1987.

16. Y. H. Lin ANd F. Y. M. WAN, Two canonical problems for the boundary conditions of axisymmetric deformation plate theories, to appear.

17. A. E. H. Love, A Treatise on the Mathematical Theory of Elasticity, 4th Ed., Dover, New York, 1944.

18. E. L. ReISS, Symmetric bending of thick circular plates, J. of S. I. A. M. 10:596-609 (1962).

19. E. L. ReISS and S. Locke, On the theory of plane stress, Quart. Appl. Math. 19:195-203 (1961).

20. E. ReIssner, On the derivation of boundary conditions for plate theory, Proc. Royal Soc. A 276:178-186 (1963).

21. S. TIMOSHENKO and N. GOODIER, Theory of Elasticity, 2nd Ed., McGraw-Hill, New York, 1951. 